\title{
Valuations and Log Canonical Thresholds
}

\author{
ZHENGYU Hu
}

\begin{abstract}
The goal of this paper is to continue the investigation of valuative quasi-plurisubharmonic functions (qpsh for short) on certain valuation spaces of a regular scheme, in line with the works [4], 5], 6] of Boucksom, Favre, Jonsson, and the works [31, 32] of Jonsson, Mustaţă. We divide this paper into two parts. In the first part we mainly discuss those valuations which compute the $\log$ canonical thresholds of qpsh functions. We expect them to be useful for the conjecture [ [31, Conjecture B] raised by Jonsson and Mustaţă. In the second part we define the restriction of a valuative qpsh function to a regular subscheme and prove a number of expected results including the restriction theorem and the inversion of adjunction. We also treat some applications in complex algebraic geometry such as extensions of pluri-canonical forms on a dlt pair under an abundance assumption.
\end{abstract}

Keywords: pluri-canonical extensions, $\log$ canonical thresholds, multiplier ideals, valuations.

\section{Introduction}

Given a smooth complex algebraic variety $X$, one can consider the space of all real valuations of the function field of $X$ centered on $X$. This is a subspace of the Berkovich space of $X$, which has recently been attracted interest for its relations to the log canonical thresholds of valuative quasiplurisubharmonics or equivalently, of graded and subadditive sequences of ideals. The study of the valuation space from this point of view was initiated by S. Boucksom, C. Favre and M. Jonsson in [4], [5], [6], and then expanded by M. Jonsson and M. Mustaţă in [31], [32]. In particular, M. Jonsson and M. Mustaţă studied a conjecture (see Conjecture 3.16) concerning the valuations that compute the log canonical threshold of a fraded sequence of ideals, and its connection to the Openness Conjecture of Demailly and Kollár on (analytic) plurisubharmonic functions. Note that Q. Guan and

Received April 21, 2012.

2010 MSC: 14F18 (Primary) 12J20 (Secondary) 
$\mathrm{X}$. Zhou announced a proof of the openness conjecture (see [27]) and that Lempert also announced a particularly simple proof (see [38]) recently.

In 29] we began the study of certain functions defined on the tempered valuation space on a regular scheme by following [31. We briefly introduce some terminology from the valuation theory. Given a regular scheme (see Section [?]), a tempered valuation $v$ is a real valuation of $K(X)$ of finite log discrepancy $A(v)<\infty$, and the tempered valuation space $\mathrm{V}_{X}$ is the subspace consisting of tempered valuations. Given a $\log$ resolution $(Y, D)$ of $X$, we can define the retraction of a valuation $v$ by setting $r_{Y, D}(v)\left(D_{i}\right)=v\left(D_{i}\right)$. In particular, a real valuation $v$ is said to be quasi-monomial if there exists a $\log$ resolution $(Y, D)$ such that $v=r_{Y, D}(v)$.

A valuative function $\varphi$ is said to be bounded homogeneous if $\varphi(t v)=$ $t \varphi(v)$ for all tempered valuations $v$ and $t \in \mathbb{R}_{+}$and if $\sup _{v \in \mathrm{V}_{X}^{*}} \frac{|\varphi(v)|}{A(v)}<\infty$. For such functions we can define the norm $\|\varphi\|=\sup _{v \in \mathrm{V}_{X}^{*}} \frac{|\varphi(v)|}{A(v)}$ on the set of bounded homogeneous functions. A typical example of such function is that one $c \log |\mathfrak{a}|$ induced from a coherent ideal sheaf (or simply an ideal) $\mathfrak{a}$ on $X$ and a positive real number $c$ by letting $c \log |\mathfrak{a}|(v)=-c v(\mathfrak{a})$. In this case the norm of the function $c \log |\mathfrak{a}|$ is nothing but the reciprocal of the log canonical threshold $\operatorname{lct}\left(\mathfrak{a}^{c}\right)$. Further, we define a valuative quasi-plurisubharmonic (qpsh for short) function to be a function that lies within the closure of the set of such functions induced by ideals. As in complex geometry we can define the multiplier ideal associated to a qpsh function. In [29] we define the multiplier ideal $\mathcal{J}(\varphi)$ of $\varphi$ to be the largest ideal $\mathfrak{a}$ satisfying that $\|\log |\mathfrak{a}|-\varphi\|<1$. One can see that this definition is reasonable (see [[29], Proposition 4.3]).

Because the $\log$ canonical threshold $\operatorname{lct}(\mathfrak{a})$ is a fundamental invariant both in singularity theory and birational geometry (see [36, 35] and etc.), we will discuss some properties of the norm of a qpsh function in detail. We say that a nontrivial tempered valuation $v$ computes $\|\varphi\|$ if the equality $\|\varphi\|=\frac{|\varphi(v)|}{A(v)}$ holds. One of the main results of [29] asserts that, for every qpsh function $\varphi$, there exists a nontrivial tempered valuation $v$ which computes $\|\varphi\|$. See also [31] and 32] for more discussions. As suggested by M. Jonsson and M. Mustaţă, the following conjecture is closely related to the Openness Conjecture in analytic geometry (see [31, Conjecture B and Theorem 7.8] and [32]). This conjecture is already known in several special cases (see [31, Section 8 and 9]).

Conjecture 1.1 (=Conjecture 3.16). If $\varphi$ is a qpsh function on $X$, then there exists a nontrivial quasi-monomial valuation $v$ which computes $\|\varphi\|$. 
Conversely, if a nontrivial tempered valuation $v$ computes the norm of some qpsh function, then $v$ is quasi-monomial.

To investigate those valuations which compute some log canonical thresholds, we first define a class of algebraic qpsh functions which can be regarded as the global version of valuative transforms of tame psh weights in [4, Section 5.3, Section 5.2]. More precisely, a qpsh function $\varphi$ is said to be tame if there exists a constant $C>0$ such that $\mathcal{J}((t+C) \varphi) \subseteq \mathfrak{a}(t \varphi)$ for every $t \geq 0$ where $\mathfrak{a}(t \varphi)$ is defined to be the largest ideal $\mathfrak{a}$ such that $\log |\mathfrak{a}| \leq \varphi$. These functions behave very nicely in many situations. An important observation is that, to every tempered valuation $v$, one can naturally associate a tame function $\phi_{v}$.

A tempered valuation $v$ is said to be computing if there exists a qpsh function $\varphi$ such that $v$ computes the norm $\|\varphi\|$. According to Lemma 3.17. this is equivalent to saying that $v$ computes the norm $\left\|\phi_{v}\right\|$ of its associate tame function.

Theorem 1.2 (=Theorem 3.25). Let $v$ be a nontrivial tempered valuation. If $r_{Y, D}(v)$ is computing for all sufficiently high log resolutions $(Y, D)$ of $X$, then $v$ is computing.

It is also natural to ask if the converse statement of the previous theorem is true, which is closely related to Conjecture 3.16 .

Conjecture 1.3 (=Conjecture 3.27). Let $v$ be a nontrivial tempered valuation. If $v$ is computing, then $r_{Y, D}(v)$ is computing for all sufficiently high log resolutions $(Y, D)$ of $X$.

Now we introduce the restriction of a qpsh function. Consider an irreducible regular closed subscheme $Z$ and a qpsh function $\varphi$. If we write $\varphi_{k}=\frac{1}{k} \log |\mathcal{J}(k \varphi)|$, then we say that $\varphi$ satisfies the restriction condition to $Z$ if

- $\varphi_{k \mid Z}:=\frac{1}{k} \log \left|\mathcal{J}(k \varphi) \cdot \mathcal{O}_{Z}\right|$ is well-defined for every integer $k$, and

- $\varphi_{k \mid Z}$ converges strongly in the norm.

In this case we define the restriction $\varphi_{\mid Z}$ to be the limit function of $\varphi_{k \mid Z}$.

Unfortunately, this definition is not a sufficiently good analogue of the restriction of qpsh function in analytic geometry via the valuative transformation (see [4,5.2] for valuative transform of a psh germ). If we take the analytic psh germ $\varphi=-\log \left(-\log \max \left|z_{1}\right|\right)$ around the origin of $\mathbb{C}^{2}$, then the valuative transform $\widehat{\varphi}$ is the zero function because the singularity is too 
mild to be detected by tempered valuations. On the other hand, the equation $\varphi(z) \equiv-\infty$ holds identically on $\left\{z_{1}=0\right\}$. See Remark 4.2 .

As in complex algebraic geometry, we conclude the following expected Restriction Theorem.

Theorem 1.4 (=Theorem 4.6). If $\varphi$ is a qpsh function which satisfies the restriction condition to $Z$, then we have the inclusion

$$
\mathcal{J}\left(\varphi_{\mid Z}\right) \subseteq \mathcal{J}(\varphi) \cdot \mathcal{O}_{Z}
$$

We can interpret the theorem above in terms of log canonical thresholds. See Definition 4.7 for the definition of local log canonical thresholds.

Theorem 1.5 (=Theorem 4.8). Let $\varphi$ be a qpsh function which satisfies the restriction condition to $Z$. Given a point $\xi \in Z$, we have the inequality of norms $\left\|\varphi_{\mid Z, \xi}\right\| \geq\left\|\varphi_{\xi}\right\|$, or equivalently of log canonical thresholds $\operatorname{lct}_{\xi}\left(\varphi_{\mid Z}\right) \leq$ $\operatorname{lct}_{\xi}(\varphi)$. In particular, given a closed subset $K$ of $Z$, we have $\operatorname{lct}_{K}\left(\varphi_{\mid Z}\right) \leq$ $\operatorname{lct}_{K}(\varphi)$.

In the theory of classification of higher dimensional varieties inversion of adjunction provides a central tool. This was originally proved by V. Shokurov in [43] in dimension 3 and generalized to all dimensions by J. Kollár in [34]. Here is a non-exhaustive list of references in recent progress: 28], 33], 44], [45, [18, 19, 2. In this paper we will prove a version of inversion of adjunction on a regular scheme.

Theorem 1.6 (=Theorem 4.12). Let $H \subseteq X$ be an irreducible regular closed subscheme of codimension one, and let $\varphi$ be a qpsh function which satisfies the restriction condition to $H$. Write $\psi=\log \left|\mathcal{O}_{X}(-H)\right|$. Given a point $\xi \in H, \operatorname{lct}_{\xi}\left(\varphi_{\mid H}\right) \geq 1$ if and only if $\operatorname{lct}_{\xi}(\varphi+\psi) \geq 1$. In particular, given a closed subset $Z$ of $H, \operatorname{lct}_{Z}\left(\varphi_{\mid H}\right) \geq 1$ if and only if $\operatorname{lct}_{Z}(\varphi+\psi) \geq 1$.

As an application to complex algebraic geometry we prove the following extension of pluri-canonical forms on a log smooth pair. See [14, Theorem 1.7] and [23, Proposition 5.11] for a comparison. Recall that the augmented base locus of a big line bundle $L$ is defined to be $\mathbf{B}_{+}(L)=\bigcap_{A} \mathbf{B}(L-A)$ where $A$ runs over all ample divisors, and that the restricted base locus of a pseudo-effective line bundle $L$ is defined to be $\mathbf{B}_{-}(L)=\bigcup_{A} \mathbf{B}(L+A)$ where $A$ runs over all ample divisors. We use the notations $v\left(\|L\|_{Z}\right):=v\left(\mathfrak{a}_{\bullet} \mid Z\right)$ where $\left\{\mathfrak{a}_{k \mid Z}=\mathfrak{b}(\lfloor k L\rfloor \mid) \cdot \mathcal{O}_{Z}\right\}$ is a graded sequence of ideals and $\sigma_{v}\left(\|L\|_{Z}\right):=$ 
$\sup _{A} v\left(\|L+A\|_{Z}\right)$ where $A$ runs over all ample divisors. See Section 5 for more details.

Let $(X, S+B)$ be a dlt pair such that $\lfloor S+B\rfloor=S$. Assume that the restricted base locus $\mathbf{B}_{-}\left(K_{X}+S+B\right)$ does not contain any lc centre of $(X, S+B)$. Let $\pi: X^{\prime} \rightarrow X$ be a $\log$ resolution of $(X, S+B)$ and we write

$$
K_{X^{\prime}}+S^{\prime}+B^{\prime}=\pi^{*}\left(K_{X}+S+B\right)+E^{\prime}
$$

where $S^{\prime}+B^{\prime} \geq 0$ and $E^{\prime} \geq 0$ do not have common components and $\left\lfloor S^{\prime}+\right.$ $\left.B^{\prime}\right\rfloor=S^{\prime}$. Following [30], [42, [14] and [10], if we consider the extension obstruction divisor

$$
\Xi:=\left.N_{\sigma}\left(\left\|K_{X^{\prime}}+S^{\prime}+B^{\prime}\right\|_{S^{\prime}}\right) \wedge B^{\prime}\right|_{S^{\prime}}
$$

on $S^{\prime}$ for every $\log$ resolution $X^{\prime}$, then we have the following result. Note that the divisor $N_{\sigma}\left(\left\|K_{X^{\prime}}+S^{\prime}+B^{\prime}\right\|_{S^{\prime}}\right)$ is well-defined on the simple normal crossing pair $\left(S^{\prime},\left.B^{\prime}\right|_{S^{\prime}}\right)$ if $\mathbf{B}_{-}\left(K_{X}+S+B\right)$ does not contain any lc centre of $(X, S+B)$ (or any stratum of the normal crossing pair $\left(S,\left.B\right|_{S}\right)$, see [[21], Section 2]).

Proposition 1.7 (=Proposition 5.18). Let $(X, S+B)$ be a dlt pair such that $\lfloor S+B\rfloor=S$. Assume that

(1). there exists an effective divisor $D \sim_{\mathbb{Q}} K_{X}+S+B$ such that $D$ contains $S$ in its support,

(2). the restricted base locus $\mathbf{B}_{-}\left(K_{X}+S+B\right)$ does not contain any $l c$ centre, and

(3). $K_{X}+S+B$ is abundant.

Let $m$ be an integer such that $m\left(K_{X}+S+B\right)$ is Cartier. If $\sigma$ is a section of $m\left(K_{S}+B_{S}\right)$ such that for every log resolution of $(X, S+B)$ we have that

$$
\operatorname{div} \pi^{*}(\sigma)+\left.m E^{\prime}\right|_{S} \geq m \Xi
$$

then $\sigma$ extends to $X$.

It is conjectured that Proposition 5.18 holds without the assumption $K_{X}+S+B$ being abundant. Using analytic methods, J.-P. Demailly, C. D. Hacon and M. Păun proved this in [14 when $(X, S+B)$ is plt and there exists an effective divisor $D \sim_{\mathbb{Q}} K_{X}+S+B$ such that $S \subseteq \operatorname{Supp}(D) \subseteq \operatorname{Supp}(S+B)$. At this point we cannot attack similar problems without using analysis. However, the analytic argument fails when $S$ has multiple components because the $L^{2}$-estimates behave badly on 
the intersection of components of $S$. It is expected that we could combine algebraic and analytic methods to deal with these problems.

Acknowledgements. This paper is based on part of the author's PhD thesis. I would like to express my deep gratitude to my supervisor Professor Kefeng Liu for numerous conversations and encouragement from him. I am indebted to Professor Hongwei Xu and many other professors in Zhejiang Unversity because I have greatly benefited from the courses and discussions with them. I would also like to thank Professor Sébastien Boucksom, Professor Mattias Jonsson and Professor Mircea Mustaţă for patiently answering my questions and providing many valuable comments. Part of this work was done when the author was visiting Professor Jungkai Chen at the Natinal Taiwan University, Taipei. I would like to thank him for his hospitality.

\section{Valuative qpsh functions}

Throughout this paper, all schemes are assumed to be separated, regular, connected and excellent noetherian schemes over $\mathbb{Q}$. All rings are assumed to be integral, regular and excellent noetherian rings containing $\mathbb{Q}$. An ideal on a scheme means a coherent ideal sheaf on a scheme. A birational model of a scheme is a scheme birational to and proper over this scheme, and a divisor over a scheme is a divisor on a birational model of the scheme. For definitions and properties of valuations, multiplier ideals, singularities in birational geometry, etc., we refer to [36, [31] and [35].

The main purpose of this section is to review [29] in which we studied certain functions defined on the tempered valuation space (see Definition 2.5 over a scheme $X$. Inside this space of functions, we isolated the closed convex cone generated by functions associated to ideals on $X$ and defined as the cone of quasi-plurisubharmonic(qpsh, for short) functions. We also introduced some basic properties of such functions.

Real valuations and quasi-monomial valuations. Let $X$ be a scheme, and let $K(X)$ be its function field. A real valuation $v$ is a function $v: K(X)^{*} \longrightarrow \mathbb{R}$ such that $v(f g)=v(f)+v(g)$ and $v(f+g) \geq$ $\min \{v(f), v(g)\}$. By convention we set $v(0):=+\infty$. Let $\mathcal{O}_{v}:=\{f \mid v(f) \geq 0\}$ be its valuation ring. If there exists a point $\xi \in X$ such that the morphism $\mathcal{O}_{X, \xi} \hookrightarrow \mathcal{O}_{v}$ is a local homomorphism, then $\xi$ is called the centre of $v$ on $X$ and denoted by $c_{X}(v)$. Note that $\xi$ is unique since $X$ is separated, and also note that the centre always exists provided that $X$ is proper over $\mathbb{Q}$. A real valuation with centered on $X$ is called a real valuation on $X$ or simply a 
valuation on $X$, and we denote by $\operatorname{Val}_{X}$ the set of valuations on $X$. The set of valuations $\mathrm{Val}_{X}$ is independent of the choice of a birational model of $X$. More precisely, if $Y \rightarrow X$ is a proper birational morphism of schemes, then $\operatorname{Val}_{X}=\operatorname{Val}_{Y}$. The trivial valuation $v$ is the valuation centered at the generic point of $X$, or equivalently, $v(f)=0$ for all $f \in K(X)^{*}$. We denote by $\mathrm{Val}_{X}^{*} \subseteq \mathrm{Val}_{X}$ the set of nontrivial valuations on $X$.

The set $\operatorname{Val}_{X}$ can be equipped with an induced topology defined by the maps $v \longrightarrow v(f)$ for all rational functions $f \in K(X)^{*}$. For every nonzero ideal $\mathfrak{a}$, recall that $v(\mathfrak{a})$ is defined as the minimum of $v(f)$ over $f \in \mathfrak{a} \cdot \mathcal{O}_{X, \xi}$ with $\xi$ the center of $v$. We have that $v(\mathfrak{a})=v(\overline{\mathfrak{a}})$ where $\overline{\mathfrak{a}}$ denotes the integral closure of $\mathfrak{a}$. Note that the topology on $\mathrm{Val}_{X}$ defined by pointwise convergence on ideals on $X$ is equivalent to that on functions in $K(X)$. Readers can consult [[31], Section 1] for more details.

For two valuations $v, w$ on $X$, we say that $v \leq w$ if $v(\mathfrak{a}) \leq w(\mathfrak{a})$ for every nonzero ideal $\mathfrak{a}$. This is equivalent to saying that the centre $\eta:=c_{X}(w) \in \overline{c_{X}(v)}$ and that $v(f) \leq w(f)$ for every nonzero local function $f \in \mathcal{O}_{X, \eta}$.

Let $\xi \in X$ be a point, and let $\underline{x}=\left(x_{1}, \ldots, x_{r}\right)$ be a regular system of parameters at $\xi$. If $f \in \mathcal{O}_{X, \xi}$ is a local regular function, then $f$ can be expressed as $f=\sum_{\beta} c_{\beta} x^{\beta}$ in $\widehat{\mathcal{O}_{X, \xi}}$ with each coefficient $c_{\beta}$ either zero or a unit. For each $\alpha=\left(\alpha_{1}, \ldots, \alpha_{r}\right) \in \mathbb{R}_{\geq 0}^{r}$, we define a real valuation by $\operatorname{val}_{\xi, \alpha}(f)=\min \left\{\langle\alpha, \beta\rangle \mid c_{\beta} \neq 0\right\}$ where $\left\langle\alpha, \beta>:=\sum_{i} \alpha_{i} \beta^{i}\right.$, which is called a monomial valuation on $X$.

A pair $(Y, D)$ is called $\log$ smooth if $Y$ is a scheme and $D$ is a reduced divisor whose components are regular subschemes intersecting each other transversally. A pair $(Y, D)$ is called a $\log$ resolution of $X$ if there is a birational projective morphism $\pi: Y \rightarrow X$ and $(Y, D+E)$ is $\log$ smooth where $E$ consists of all exceptional divisors over $X$. Let $\left(Y^{\prime}, D^{\prime}\right)$ be another log resolution of $X$, we say $\left(Y^{\prime}, D^{\prime}\right) \succeq(Y, D)$ if $Y^{\prime}$ is projective over $Y$ and the support of $D^{\prime}$ contains the support of the pull-back of $D$. Note that log resolutions of $X$ form an inverse system.

Let $(Y, D)$ be a $\log$ resolution of $X$, and let $\eta$ be the generic point of an irreducible component of the intersection of some prime components of $D$. We denote by $\mathrm{QM}_{\eta}(Y, D)$ the set of real valuations which can be defined as a monomial valuation at $\eta$. Note that $\eta \in \overline{c_{X}(v)}$ and $\operatorname{QM}_{\eta}(Y, D) \cong \mathbb{R}^{r}$ as topological spaces. We also define $\mathrm{QM}(Y, D)=\bigcup_{\eta} \mathrm{QM}_{\eta}(Y, D)$ where $\eta$ runs over every generic point of some component of the intersection of some 
prime components of $D$. A real valuation $v$ is said to be quasi-monomial if there exists a log resolution $(Y, D)$ such that $v \in \mathrm{QM}(Y, D)$.

Let $v \in \mathrm{Val}_{X}$ be a quasi-monomial valuation. A log smooth pair $(Y, D)$ is said to be adapted to $v$ if $v \in \mathrm{QM}(Y, D)$. We say that $(Y, D)$ is a good pair adapted to $v$ if $\left\{v\left(D_{i}\right) \mid v\left(D_{i}\right)>0\right\}$ are rationally independent. The following useful lemma is established as [[31], Lemma 3.6].

Lemma 2.1. Let $v \in \mathrm{Val}_{X}$ be a quasi-monomial valuation. There exists a good pair $(Y, D)$ adapted to $v$. If $\left(Y^{\prime}, D^{\prime}\right) \succeq(Y, D)$ and $(Y, D)$ is a good pair adapted to $v$, then $\left(Y^{\prime}, D^{\prime}\right)$ is also a good pair adapted to $v$.

An important class of valuations are divisorial valuations. A valuation is called divisorial if it is positively proportional to $\operatorname{ord}_{E}$ for some prime divisor $E$ over $X$, where $\operatorname{ord}_{E}$ is the vanishing order along $E$. One easily verifies that the trivial valuation is quasi-monomial of rational rank zero, and a divisorial valuation is quasi-monomial of rational rank one. Let $(Y, D)$ be a $\log$ smooth pair adapted to $v$. It can be verified that $v$ is divisorial if and only if $\mathbb{R}_{\geq 0}[v] \subseteq \mathrm{QM}_{\eta}(Y, D) \cong \mathbb{R}_{\geq 0}^{r}$ is a rational ray, that is, $\mathbb{R}_{\geq 0}[v]$ contains some rational point in $\mathbb{R}_{>0}^{r}$.

For every $\log$ resolution $(Y, D)$ we can define the retraction map

$$
r_{Y, D}: \operatorname{Val}_{X} \longrightarrow \mathrm{QM}(Y, D)
$$

by taking $v$ to a quasi-monomial valuation in $\mathrm{QM}(Y, D)$ with $r_{Y, D}(v)\left(D_{i}\right)=$ $v\left(D_{i}\right)$. Note that $r_{Y, D}$ is continuous and $v \geq r_{Y, D}(v)$ with equality if and only if $v \in \operatorname{QM}(Y, D)$. Furthermore, if $\left(Y^{\prime}, D^{\prime}\right) \succeq(Y, D)$ is another resolution, then the retraction map $r_{Y, D}: \mathrm{QM}\left(Y^{\prime}, D^{\prime}\right) \longrightarrow \mathrm{QM}(Y, D)$ (by abuse of notation if without confusion) is a surjective mapping which is integral linear on every $\mathrm{QM}_{\eta^{\prime}}\left(Y^{\prime}, D^{\prime}\right)$ and we have that $r_{Y, D} \circ r_{Y^{\prime}, D^{\prime}}=r_{Y, D}$. Therefore we can naturally regard $\mathrm{QM}(Y, D)$ as a subset of $\mathrm{QM}\left(Y^{\prime}, D^{\prime}\right)$, and hence of the set of quasi-monomial valuations on $X$. Also note that $v(\mathfrak{a}) \geq r_{Y, D}(v)(\mathfrak{a})$ for an ideal $\mathfrak{a}$ on $X$, with equality if $(Y, D)$ is a $\log$ resolution of $\mathfrak{a}$ (see [[31], Corollary 4.8]).

Tempered valuations and the tempered valuation space. We first introduce the log discrepancy on a scheme. Let $\pi: Y \longrightarrow X$ be a birational proper morphism. The $0^{\text {th }}$ fitting ideal $\operatorname{Fitt}_{0}\left(\Omega_{Y / X}\right)$ is a locally principle ideal with its corresponding effective divisor denoted by $K_{Y / X}$ (see [[31, Section 1.3]). For a quasi-monomial valuation $v \in \mathrm{QM}(Y, D)$, we define the 
$\log$ discrepancy

$$
A_{X}(v)=\sum v\left(D_{i}\right) \cdot A_{X}\left(\operatorname{ord}_{D_{i}}\right)=\sum v\left(D_{i}\right) \cdot\left(1+\operatorname{ord}_{D_{i}}\left(K_{Y / X}\right)\right) .
$$

We simply denote this by $A$ when the scheme $X$ is obvious. Note that $A$ is strictly positive linear on every $\mathrm{QM}_{\eta}(Y, D)$, and in particular continuous on every $\mathrm{QM}_{\eta}(Y, D)$. One important property of $A$ is its monotonicity with respect to retractions, that is, if $\left(Y^{\prime}, D^{\prime}\right) \succeq(Y, D)$ and $v \in \mathrm{QM}\left(Y^{\prime}, D^{\prime}\right)$, then $A(v) \geq A\left(r_{Y, D}(v)\right)$ and equality holds only when $v \in \mathrm{QM}(Y, D)$. See [[39], 41]] for the construction of a related "log discrepancy like" function, and 46. for a vast generalization. For an arbitrary valuation $v \in \mathrm{Val}_{X}$, we define

$$
A(v)=\sup _{(Y, D)} A\left(r_{Y, D}(v)\right) \in[0,+\infty] .
$$

Note that $A$ is lower-semicontinuous (lsc) as a valuative function.

Definition 2.2. A valuation $v$ is said to be tempered if $A(v)<\infty$. The tempered valuation space $\mathrm{V}_{X}$ of $X$ is defined to be the space of tempered valuations as a subspace of $\mathrm{Val}_{X}$.

We similarly denote by $\mathrm{V}_{X}^{*}$ the subset of nontrivial tempered valuations. If $f: X^{\prime} \rightarrow X$ is a proper birational morphism, then $A_{X}(v)=$ $A_{X^{\prime}}(v)+v\left(K_{X^{\prime} / X}\right)$ (see [[31], Proposition 5.1(3)]) and hence $\mathrm{V}_{X^{\prime}}=\mathrm{V}_{X}$. Since $\mathrm{V}_{X}$ is a topological subspace of $\mathrm{Val}_{X}$, it is naturally a subspace of the Berkovich space $X^{a n}$. See [31], Section 6.3] for a comparison.

With the aid of the log discrepancy, we can normalize $\mathrm{V}_{X}^{*}$ by letting $A(v)=1$, that is, we define $\Lambda_{X}:=\left\{v \in \mathrm{V}_{X}^{*} \mid A(v)=1\right\}$. In particular, we normalize every cone complex $\operatorname{QM}(Y, D)$ by setting $\Delta(Y, D):=\{v \in$ $\mathrm{QM}(Y, D) \mid A(v)=1\}$. It is clear that $\Delta(Y, D)$ naturally possess the structure of a simplicial complex, and by convention we say that $\Delta(Y, D)$ is a dual complex. Readers can compare the constructions here with [4], [5] and [6].

The following lemma allows us to compare $v$ and $\operatorname{ord}_{\xi}$ where $\xi=c_{X}(v)$ which is quite useful (see [36], [[31], Section 5.3] for the definition of $\operatorname{ord}_{\xi}$ ). See [[31], Proposition 5.10] for a proof. Recently S. Boucksom, C. Favre and M. Jonsson gave a refinement of the following lemma in [7].

Lemma 2.3 (Izumi type inequality). Let $\xi=c_{X}(v)$ and $\mathfrak{m}_{\xi}$ be the defining ideal of $\overline{\{\xi\}}$. Then, we have $v\left(\mathfrak{m}_{\xi}\right) \operatorname{ord}_{\xi} \leq v \leq A(v) \operatorname{ord}_{\xi}$.

Valuative qpsh functions. Let $X$ be a scheme and $\mathrm{V}_{X}$ be its tempered valuation space. A valuative function $\varphi$ is said to be homogeneous 
if $\varphi(t v)=t \varphi(v)$ for all $v \in \mathrm{V}_{X}$ and $t \in \mathbb{R}_{+}$. A valuative function $\varphi$ is said to be bounded if $\sup _{v \in \mathrm{V}_{X}^{*}} \frac{|\varphi(v)|}{A(v)}<\infty$. In other words, a bounded valuative function is just a bounded function on $\Lambda_{X}$. The set of bounded homogeneous functions forms an $\mathbb{R}$-linear space, which can be equipped with the norm $\|\varphi\|=\sup _{v \in \mathrm{V}_{X}^{*}} \frac{|\varphi(v)|}{A(v)}$, and will be denoted by $\mathrm{BH}(X)$. If $\mathfrak{q}$ is a nonzero ideal on $X$, then we define the $\mathfrak{q}$-norm to be $\|\varphi\|_{\mathfrak{q}}=\sup _{v \in \mathrm{V}_{X}^{*}} \frac{|\varphi(v)|}{A(v)+v(\mathfrak{q})}$. When equipped with the norm, $\mathrm{BH}(X)$ is actually a Banach space (see [[29], Proposition 3.2]).

We also define

$$
\|\varphi\|_{\mathfrak{q}}^{+}:=\sup _{v \in \mathrm{V}_{X}^{*}} \frac{\varphi(v)}{A(v)+v(\mathfrak{q})}
$$

and

$$
\|\varphi\|_{\mathfrak{q}}^{-}:=\sup _{v \in \mathrm{V}_{X}^{*}} \frac{-\varphi(v)}{A(v)+v(\mathfrak{q})} .
$$

Clearly, $\|\varphi\|_{\mathfrak{q}}^{+}=\|-\varphi\|_{\mathfrak{q}}^{-}$and $\|\cdot\|_{\mathfrak{q}}=\max \left\{\|\cdot\|_{\mathfrak{q}}^{+},\|\cdot\|_{\mathfrak{q}}^{-}\right\}$.

Given a nonzero ideal $\mathfrak{a}$, we define $|\mathfrak{a}|(v)=-e^{v(\mathfrak{a})}$ by convention. It is obvious that $\log |\mathfrak{a}|$ is a continuous bounded homogeneous function.

Definition 2.4. A bounded homogeneous function $\varphi$ is said to be an ideal function if there exists a finite number of nonzero ideals $\mathfrak{a}_{j}$ and positive real numbers $c_{j}$ such that $\varphi=\sum_{j=1}^{l} c_{j} \log \left|\mathfrak{a}_{j}\right|$.

Definition 2.5. A bounded homogeneous function $\varphi$ is said to be a valuative quasi-plurisubharmonic (qpsh for short) function if there exists a sequence of ideal functions which converges to $\varphi$ strongly in the norm. The set of valuative qpsh functions, which is a closed convex cone in $\operatorname{BH}(X)$, is denoted by $\mathrm{QPSH}(X)$. We usually omit "valuative" if there is no confusion.

Readers can compare the constructions here with [4]. If we work on $X=\operatorname{Spec} \widehat{R}$ where $R$ is the localization of $\mathbb{C}\left[x_{1}, \ldots, x_{n}\right]$ at the origin, then our definition of qpsh functions coincides the notion of formal psh functions. A brief argument is as follows. Given a formal psh function $g$, we have a subadditive sequence of ideals $\left\{\mathcal{L}^{2}(t g)\right\}_{t>0}$ in $\widehat{R}$ by [4], Theorem 3.10] which satisfies that $v\left(\mathcal{L}^{2}(t g)\right)+A(v)+(1+\epsilon) \operatorname{tg}(v) \geq 0$ for every quasi-monomial valuation $v$ centered at the origin and an arbitrary small $\epsilon=\epsilon(t)$ depending on $t$ by [4], Theorem 3.9]. It follows that $\left\{\mathcal{L}^{2}(t g)\right\}_{t>0}$ form a subadditive sequence of ideals of controlled growth which induces a qpsh function $\varphi$ on $X$ by definition (see [[31], Definition 2.9] for the definition of "controlled growth"). Therefore $\varphi(v)=g(v)$ for every divisorial valuation $v$ centered 
at the origin. Conversely, a qpsh function can be naturally viewed as a formal psh function by definition. Therefore we constructed an one-to-one correspondence.

Remark 2.6. Recall from complex geometry that a function $\varphi: X \rightarrow$ $[-\infty,+\infty)$ from a complex manifold is qpsh if it is locally equal to the sum of a smooth function and a psh function. If $X$ is a smooth complex variety, then we should be able to define the valuative transform of $\varphi$ which is expected to be a qpsh function on the tempered valuation space $\mathrm{V}_{X}$ as defined in this paper. This was done locally in [4] and its predecessors [24], [25], [26]. However, the global situation is not fully understood by us at this point.

We can also define the multiplier ideal of a (valuative) qpsh function as in complex analysis and geometry,

Definition 2.7. For a qpsh function $\varphi \in \mathrm{BH}(X)$, the multiplier ideal $\mathcal{J}(\varphi)$ of $\varphi$ is defined to be the largest ideal in the set of nonzero ideals $\{\mathfrak{a}|\| \log | \mathfrak{a} \mid-$ $\left.\varphi \|^{+}<1\right\}$.

If $\varphi$ is of the form $\sum_{i=1}^{l} c_{i} \log \left|\mathfrak{a}_{i}\right|$, then $\mathcal{J}(\varphi)=\mathcal{J}\left(\prod_{i=1}^{l} \mathfrak{a}_{\mathfrak{i}}{ }^{c_{i}}\right)$. Therefore the definition given by valuative analysis coincides with the classic algebraic definition of multiplier ideals. See [[29], Proposition 4.3].

The first important property of a qpsh function is that it is a decreasing limit of a sequence of qpsh functions of the form $c_{k} \log \left|\mathfrak{b}_{k}\right|$. In complex analysis and geometry, such a regularization is crucial. See [11, [12]. Moreover, we can actually choose $\mathfrak{b}_{k}=\mathcal{J}(k \varphi)$ where $\mathcal{J}(k \varphi)$ satisfies the subadditivity property (See [13] for a comparison). For the definition and basic properties of subadditive sequence of ideals (of controlled growth), we refer to [31]. Readers could see [[29], Theorem 4.24] for a proof.

Theorem 2.8. Let $\varphi$ be a bounded homogeneous function. Then $\varphi$ is qpsh if and only if $\varphi$ is the limit function, in the norm, of a decreasing sequence of qpsh functions of the form $c_{k} \log \left|\mathfrak{b}_{k}\right|$. Furthermore, we can choose $c_{k}=\frac{1}{k}$ and $\mathfrak{b}_{k}=\mathcal{J}(k \varphi)$ which form a subadditive sequence of ideals.

Note that a pointwise limit of a decreasing sequence of qpsh functions of the form $c_{k} \log \left|\mathfrak{b}_{k}\right|$ is not necessarily qpsh.

Example 2.9. Let $X=$ Spec $k[x]$ be an affine line, and let $\phi_{p}=$ $\sum_{j=1}^{p} \log \left|f_{j}\right|$ where $f_{j}=x-j$. We see that $\phi_{p}$ is a decreasing sequence of 
ideal functions and the pointwise limit function $\varphi$ exists. But $\varphi$ is not qpsh because $\|\varphi-\phi\| \geq 1$ for any ideal function $\phi$.

Now we define a set of qpsh functions associated to a graded sequence of ideals. For the definition and basic properties of a graded sequence of ideals, please refer to 31. See also [29]. For a graded sequence of ideals $\mathfrak{a}_{\bullet}$, we use the notation $\log \left|\mathfrak{a}_{\bullet}\right|$ to indicate the limit function of $\frac{1}{k} \log \left|\mathfrak{a}_{k}\right|$.

Definition 2.10. A qpsh function $\varphi$ is algebraic if it is the poinwise limit function of an increasing sequence of ideal functions $\varphi=\lim _{m \rightarrow \infty} \varphi_{m}$. Equivalently, a qpsh function $\varphi$ is algebraic if it can be written as $\varphi=\log \left|\mathfrak{a}_{\bullet}\right|$ which is associated to a graded sequence of ideals $\mathfrak{a}_{\bullet}$. The set of algebraic qpsh functions is denoted by $\operatorname{QPSH}^{a}(X)$.

Note that a general qpsh function is not necessarily algebraic.

Example 2.11. Let $X=\operatorname{Spec} k\left[x_{1}, x_{2}\right]$ be the affine plane. If we set $\phi_{k}=$ $\sum_{l=1}^{k} \frac{1}{2^{l}} \log \left|f_{l}\right|$ where $f_{l}=x_{1}+x_{2}^{2^{l}}$, then $\phi_{k}$ converges to a qpsh function $\phi$ strongly in the norm. However, the qpsh function $\phi$ is not algebraic because there is no ideal function $\varphi \leq \phi$.

In fact we can choose the standard graded sequence of ideals associated to an algebraic qpsh function. Before that we introduce the notion of envelope ideals.

Definition 2.12. Let $\varphi \in \mathrm{BH}(X)$ be a bounded homogeneous function. Its envelope ideal $\mathfrak{a}(\varphi)$ is defined to be the largest ideal in the set $\{\mathfrak{a}|\log | \mathfrak{a} \mid \leq \varphi\}$ if this set is nonempty. If it is empty, we set $\mathfrak{a}(\varphi)=0$.

Note that the envelope ideal of an algebraic qpsh function is always nonzero. If we set $\mathfrak{a}(\varphi)_{m}=\mathfrak{a}(m \varphi)$, then $\{\mathfrak{a}(\varphi) \bullet\}$ is a graded sequence of ideals. We can easily show that every algebraic qpsh function is of the form $\log |\mathfrak{a}(\varphi) \cdot|$. (See [[29], Theorem 4.12]).

Also note if an algebraic qpsh function $\varphi$ is associated to a graded sequence of ideals $\mathfrak{a}_{\bullet}$, then its multiplier ideal $\mathcal{J}(\varphi)=\mathcal{J}\left(\mathfrak{a}_{\bullet}\right)$. Therefore the definition from valuative analysis is compatible with the algebraic definition of multiplier ideals of graded sequences of ideals. See [[29], Corollary 4.14]. 


\section{Log canonical thresholds}

Given an ideal $\mathfrak{a}$ on a scheme $X$, the $\log$ canonical threshold $\operatorname{lct}(\mathfrak{a})$ is a fundamental invariant both in singularity theory and birational geometry (see [35], [36] and etc.). The log canonical threshold admits the following description in terms of valuations:

$$
\operatorname{lct}(\mathfrak{a})=\inf _{E} \frac{A\left(\operatorname{ord}_{E}\right)}{\operatorname{ord}_{E}(\mathfrak{a})}
$$

where $E$ runs over all prime divisors over $X$ and $A\left(\operatorname{ord}_{E}\right)=\operatorname{ord}_{E}\left(K_{Y / X}\right)+1$. In fact in the above formulae one can take the infimum over all real valuations centered on $X$. It is well-known that if $Y$ is a $\log$ resolution of $\mathfrak{a}$, then there exists some prime divisor $E$ on $Y$ such that $\operatorname{ord}_{E}$ computes the log canonical threshold, that is, $\operatorname{lct}(\mathfrak{a})=\frac{A\left(\operatorname{ord}_{E}\right)}{\operatorname{ord}_{E}(\mathfrak{a})}$. Given a qpsh function $\varphi$, we can define the log canonical threshold as

$$
\operatorname{lct}(\varphi)=\inf _{E} \frac{A\left(\operatorname{ord}_{E}\right)}{-\varphi\left(\operatorname{ord}_{E}\right)}
$$

We can show that $\operatorname{lct}(\varphi)$ equals to the limit of $\frac{1}{c_{k}} \operatorname{lct}\left(\mathfrak{a}_{k}\right)$ where $c_{k} \log \left|\mathfrak{a}_{k}\right|$ converges to $\varphi$ strongly in the norm (see [[29], Theorem 4.24, Remark 4.25] for an argument). Unfortunately, there may be no divisorial valuation which computes the log canonical threshold in general. However, we can prove that there exists a tempered valuation which computes the log canonical threshold. This has been thoroughly studied in [31], [32] and other references. It is conjectured (see [31], Conjecture B]) that a valuation which computes the $\log$ canonical threshold is always quasi-monomial. Equivalently we consider the reciprocal of the log canonical threshold which is exactly the norm of $\varphi$ by definition as below. By definition we note that $\operatorname{lct}(\varphi)^{-1}=\|\varphi\|^{+}$which is also known as the Arnold multiplicity in the literature.

Definition 3.1. Let $\varphi$ be a bounded homogeneous function and $\mathfrak{q}$ be a nonzero ideal on $X$. We say a nontrivial tempered valuation $v \in \mathrm{V}_{X}^{*}$ computes $\|\varphi\|_{\mathfrak{q}}$ if the equality $\|\varphi\|_{\mathfrak{q}}=\frac{|\varphi(v)|}{A(v)+v(\mathfrak{q})}$ holds.

More generally, for a nonzero ideal $\mathfrak{q}$, there exists a tempered valuation which compute this norm.

Theorem 3.2. Let $\varphi$ be a qpsh function and let $\mathfrak{q}$ be a nonzero ideal on $X$. Then there exists a nontrivial tempered valuation $v$ which computes $\|\varphi\|_{\mathfrak{q}}$. 
Readers can consult [29] for a proof. Also see [31] and [32] for more discussions.

Tame qpsh functions. We will discuss a class of algebraic qpsh functions whose associated graded sequence of envelope ideals and associated subadditive sequence of multiplier ideals "converge uniformly" by abuse of language (see Remark 3.10. If we work on $X=\operatorname{Spec} \widehat{R}$ where $R$ is the localization of $\mathbb{C}\left[x_{1}, \ldots, x_{n}\right]$ at the origin, then these tame qpsh functions defined below are very close to the valuative transforms of tame psh weights in [4], Section 5.3, Section 5.2].

Definition 3.3. Let $\varphi$ be a qpsh function on $X$. A qpsh $\varphi$ is said to be tame if there exists a constant $C>0$ such that

$$
\mathcal{J}((t+C) \varphi) \subseteq \mathfrak{a}(t \varphi)
$$

for every $t \geq 0$. Such a constant can be chosen to be minimal, and is called its tameness constant. A qpsh function $\varphi$ is said to be weakly tame if there exists a nonzero ideal $\mathfrak{c}$ such that

$$
\mathcal{J}(t \varphi) \cdot \mathfrak{c} \subseteq \mathfrak{a}(t \varphi)
$$

for all sufficiently large numbers $t \gg 0$. Such an ideal can be chosen to be maximal, and is called its tameness ideal.

Example 3.4. Given a complex projective manifold $X$ and a big line bundle $L$, we have a graded sequence of ideals $\mathfrak{a}_{\bullet}:=\{\mathfrak{b}(|m L|)\}_{m=1}^{\infty}$, where $\mathfrak{b}(|m L|)$ denotes the base ideal of $m L$, and we have the corresponding algebraic qpsh function $\phi=\log \left|\mathfrak{a}_{\bullet}\right|$. Then, $\phi$ is weakly tame according to [[36], Theorem 11.2.21].

Lemma 3.5. (1). If $\varphi$ is tame, then it is weakly tame and its tameness ideal contains $\mathfrak{a}(\eta \varphi)$ where $\eta$ is its tameness constant.

(2). If $\varphi$ is (weakly) tame, then so is t $\varphi$ for any $t>0$. If $\varphi$ and $\psi$ are both (weakly) tame, then so is $\varphi+\psi$.

(3). A (weakly) tame function $\varphi$ is algebraic.

Proof. (1). The assertion directly follows from the inclusion $\mathcal{J}(t \varphi) \cdot \mathfrak{a}(\eta \varphi) \subseteq$ $\mathfrak{a}((t-\eta) \varphi) \cdot \mathfrak{a}(\eta \varphi) \subseteq \mathfrak{a}(t \varphi)$ for $t \geq \eta$.

(2). We only prove the case when $\varphi$ and $\psi$ are weakly tame here because the tame case can be proved in a similar way. If $\varphi$ is weakly tame, then 
$\mathcal{J}\left(t^{\prime} t \varphi\right) \cdot \mathfrak{c} \subseteq \mathfrak{a}\left(t^{\prime} t \varphi\right)$ for every sufficiently large number $t^{\prime}$. If $\varphi$ and $\psi$ are weakly tame with their tame ideals $\mathfrak{c}$ and $\mathfrak{c}^{\prime}$ respectively, then

$$
\mathcal{J}(t(\varphi+\psi)) \cdot \mathfrak{c} \cdot \mathfrak{c}^{\prime} \subseteq \mathcal{J}\left(t ( \varphi ) \cdot \mathfrak { c } \cdot \mathcal { J } \left(t(\psi) \cdot \mathfrak{c}^{\prime} \subseteq \mathfrak{a}(t \varphi) \cdot \mathfrak{a}(t \psi) \subseteq \mathfrak{a}(t(\varphi+\psi))\right.\right.
$$

for every sufficiently large number $t$.

(3). If $\varphi$ is weakly tame, then $\left\|\frac{1}{m} \log |\mathcal{J}(m \varphi)|-\frac{1}{m} \log |\mathfrak{a}(m \varphi)|\right\| \leq$ $\frac{1}{m}\|\log |\mathfrak{c}|\|$. Therefore $\frac{1}{m} \log |\mathfrak{a}(m \varphi)|$ converges to $\varphi$ strongly in the norm.

Proposition 3.6. If $\varphi$ is tame with its tameness constant $\eta$, then $\eta \geq$ $\operatorname{lct}(\varphi)$.

Proof. If $\eta<\lambda:=\operatorname{lct}(\varphi)$, then one has

$$
\mathcal{O}_{X}=\mathcal{J}((\eta+\varepsilon) \varphi) \subseteq \mathcal{I}(\varepsilon \varphi) \neq \mathcal{O}_{X}
$$

for $\varepsilon$ sufficiently small, which is a contradiction.

Now we need the notion of the envelope qpsh function of a bounded homogeneous function.

Definition 3.7. Let $\varphi$ be a bounded homogeneous function. Assume that the set $\{\psi \in \operatorname{QPSH}(X) \mid \psi \leq \varphi\}$ is nonempty. Then we say the maximal function in this set the qpsh envelope function. The existence of such maximal function follows from [[29], Lemma 4.15 and Definition 4.16] We similarly define the algebraic qpsh envelope function of $\varphi$ if it exists. Also note that the algebraic qpsh envelope function exists if and only if the set $\left\{\psi \in \operatorname{QPSH}^{a}(X) \mid \psi \leq \varphi\right\}$ is nonempty.

Lemma 3.8. Let $\varphi$ be a bounded homogeneous function. If $\varphi$ is determined on some dual complex $\Delta(Y, D)$ in the sense of $\varphi=\varphi \circ r_{Y, D}$, and if we denote the image of $D$ on $X$ by $Z$, then its qpsh envelope function $\psi$ exists and it is weakly tame. Assume further that $\inf _{v \in V_{X, \xi}} \varphi(v)<0$ for every generic point $\xi$ of $Z$. Then $\psi$ is tame.

Proof. The existence of $\psi$ follows from [29], Lemma 4.17]. Let $\mathfrak{m}$ be the corresponding ideal of $Z$. Note that $\log |\mathfrak{m}|$ is strictly negative on $\Delta(Y, D)$. There exists an integer $k$ such that $v\left(\mathfrak{m}^{k}\right) \geq 1$ on $\Delta(Y, D)$. If $f \in \Gamma(U, \mathcal{J}(t \psi))$, then $v(f)+A(v)+t \psi(v)>0$ for every $v$ centered on $U$. The inequality $v(f)+v\left(\mathfrak{m}^{k}\right)+t \varphi(v)>0$ holds for every valuation $v$ in $\Delta(Y, D)$ centered on $U$, hence it holds on $V_{U}$ by the convexity of $\log |f|+k \log |\mathfrak{m}|$ (see [[29], Proposition 3.11]). It follows that $\mathcal{J}(t \psi) \cdot \mathfrak{m}^{k} \subseteq \mathfrak{a}(t \varphi)=\mathfrak{a}(t \psi)$. 
If $\inf _{v \in V_{X, \xi}} \varphi(v)<0$ for every generic point $\xi$ of $Z$, then $\psi$ is strictly negative on $\Delta(Y, D)$. Therefore there exists a positive number $C$ such that $C \psi(v)<-1$ for every valuation $v$ in $\Delta(Y, D)$. For any $f \in \Gamma(U, \mathcal{J}((t+$ $C) \psi)$ ), we have $v(f)+t \psi(v)>0$ for every $v$ in $\Delta(Y, D)$ centered on $U$. Since $\psi \leq \varphi$, we have that $v(f)+t \varphi(v)>0$ holds for every $v$ in $\Delta(Y, D)$ centered on $U$ and hence holds for every $v \in V_{U}$. It follows that $f \in \mathfrak{a}(t \psi)$ which implies the conclusion.

An immediate consequence of the previous lemma is the following corollary.

Corollary 3.9. Every ideal function is tame.

Remark 3.10. If $\varphi$ is weakly tame with its tameness ideal $\mathfrak{c}$, then we have

$$
\mathfrak{a}(m l \varphi) \cdot \mathfrak{c}^{l} \subseteq \mathcal{J}(m l \varphi) \cdot \mathfrak{c}^{l} \subseteq \mathcal{J}(m \varphi)^{l} \cdot \mathfrak{c}^{l} \subseteq \mathfrak{a}(m \varphi)^{l}
$$

for every sufficiently large integer $m$ and every integer $l$. In particular, $\varphi+\frac{1}{k} \log |\mathfrak{c}| \leq \frac{1}{k} \log |\mathfrak{a}(k \varphi)|$ for every sufficiently large integer $k$. However, we still have no idea if the converse would be true (possibly with some extra assumption).

On the other hand, we have

$$
\mathcal{J}(m \varphi)^{l} \cdot \mathfrak{c}^{l} \subseteq \mathfrak{a}(m \varphi)^{l} \subseteq \mathfrak{a}(m l \varphi) \subseteq \mathcal{J}(m l \varphi)
$$

for every sufficiently large integer $m$ and every integer $l$. In particular, $\varphi \leq$ $\frac{1}{k} \log |\mathcal{J}(k \varphi)|+\frac{1}{k} \log |\mathfrak{c}|$ for every sufficiently large integer $k$. Conversely, such an inequality implies that $\varphi$ is weakly tame by the definitions of envelope ideals and weak tameness.

Tame Functions associated to Tempered Valuations. To investigate more on the structure of the valuation space, we observe that for every nontrivial tempered valuation $v$, we can construct the corresponding tame function in a natural way.

Definition 3.11. Let $v \in V_{X}^{*}$ be a nontrivial tempered valuation. We define a valuative function $d_{v}(w)=\left\{\begin{array}{ll}1 & \text { if } w=v \\ 0 & \text { otherwise }\end{array}\right.$ which is called the Dirac function of $v$. Further, we define $\phi_{v}$ to be the qpsh envelope function of $-d_{v}$.

In fact we can describe the function $\phi_{v}$ more explicitly. 
Lemma 3.12. (1). $\Gamma\left(U, \mathfrak{a}\left(t \phi_{v}\right)\right)=\left\{f \in \mathcal{O}_{X}(U) \mid v(f) \geq t\right\}$ if $c_{X}(v) \in U$ and it is trivial otherwise. In particular, $\phi_{v}(v)=-1$.

(2). $\phi_{v}$ is tame with its tameness constant $\eta_{v} \leq A(v)$.

(3). $\phi_{v}(w)=-\inf _{\mathfrak{b}} \frac{w(\mathfrak{b})}{v(\mathfrak{b})}$ where $\mathfrak{b}$ runs over all nonzero ideals such that $v(\mathfrak{b}) \neq$ 0 .

Proof. (1). Let $\mathcal{I}$ be an ideal sheaf such that $\Gamma(U, \mathcal{I})=\left\{f \in \mathcal{O}_{U} \mid v(f) \geq t\right\}$ if $c_{X}(v) \in U$ and trivial otherwise. We have that $\mathfrak{a}\left(t \phi_{v}\right) \subseteq \mathcal{I}$ and equality holds if $\mathcal{I}$ is coherent. By a similar argument used in the proof of [[29], Proposition 4.10] we obtain the conclusion. Consequenctly $\phi_{v}(v)=-1$ by [[31], Lemma $2.4]$.

(2). Let $C=A(v)$. If $f \in \Gamma\left(U, \mathcal{J}\left((t+C) \phi_{v}\right)\right)$, then $w(f)+A(w)+(t+$ $C) \phi_{v}(w)>0$ for every $w \in V_{U}$, and it follows that $v(f)>t$ when $c_{X}(v) \in U$. Therefore $f \in \Gamma\left(U, \mathfrak{a}\left(t \phi_{v}\right)\right)$.

(3). Since $\phi_{v}$ is tame, it is algebraic according to Lemma 3.5. Therefore, $\phi_{v}=\log \left|\mathfrak{a}\left(\phi_{v}\right)_{\bullet}\right|$. Now we apply [31], Lemma 2.4] and obtain the conclusion.

An important feature of a qpsh function is its support. We introduce the definition of the support of a qpsh function from [[29], Definition 3.10].

Definition 3.13. The support of a qpsh function $\varphi$ is defined to be the set $\left\{x \in X \mid x=c_{X}(v)\right.$ for some nontrivial tempered valuation $v$ such that $\varphi(v)<0\}$, and is denoted by $\operatorname{Supp}(\varphi)$.

We have a very straightforward description of the support of a tame function $\phi_{v}$ as below.

Corollary 3.14. $\operatorname{Supp}\left(\phi_{v}\right)=\overline{\left\{c_{X}(v)\right\}}$.

Proof. If $\phi_{v}(w)>0$, then by Lemma 3.12(3) we have $t w \geq v$ for some real number $t>0$. Hence $c_{X}(w)=c_{X}(t w) \in\left\{c_{X}(v)\right\}$.

Another feature of $\phi_{v}$ is that the mapping $v \rightarrow \phi_{v}$ preserves the partial order of tempered valuations.

Proposition 3.15. $w \geq v$ if and only if $\phi_{w} \geq \phi_{v}$.

Proof. If $w \geq v$, then $\phi_{v}(w) \leq \phi_{v}(v)=-1=\phi_{w}(w)$ by Lemma 3.12(1). Since $\phi_{w}$ is the qpsh envelope function of $-d_{w}$, the inequality $\phi_{w} \geq \phi_{v}$ follows directly by definition. Conversely, if $\phi_{w} \geq \phi_{v}$, then $-1=\phi_{w}(w) \geq \phi_{v}(w)$. Thus $w \geq v$ by Lemma $3.12(3)$. 
Computing Sets of Log Canonical Thresholds. We continue our study by computing the log canonical thresholds of qpsh functions, or equivalently by computing the norms (see [[29], Section 5]). The following conjecture was raised as [[31], Conjecture B] (see also [[31, Theorem 7.8]). It is already known for several special cases (see [[31, Section 8 and 9] for thorough proofs). Their recent work 32 reveals the relation between this conjecture and the openness conjecture. Recently Q. Guan and X. Zhou announced a proof of the openness conjecture (see [27]).

Conjecture 3.16. Let $\varphi$ be a qpsh function on $X$ and $\mathfrak{q}$ be a nonzero ideal on $X$. Then there exists a nontrivial quasi-monomial valuation $v$ which computes $\|\varphi\|_{\mathfrak{q}}$. Conversely, if a nontrivial tempered valuation $v$ computes the norm of some qpsh function, then $v$ is quasi-monomial.

Further, one can ask if it is possible to characterize those tempered valuations which compute the norm of some qpsh function. By convention we use the notation $\operatorname{lct}^{\mathfrak{q}}(\varphi)$ as the reciprocal of $\|\varphi\|_{\mathfrak{q}}$. The following lemma and its proof are taken from [31].

Lemma 3.17. Let $v \in V_{X}^{*}$ be a nontrivial tempered valuation. The following statements are equivalent.

(1). There exists a qpsh function $\varphi$ such that $v$ computes $\operatorname{lct}^{\mathfrak{q}}(\varphi)$.

(2). For every tempered valuation $w \geq v$, we have $A(w)+w(\mathfrak{q}) \geq A(v)+$ $v(\mathfrak{q})$.

(3). $v$ computes $\operatorname{lct}^{\mathfrak{q}}\left(\phi_{v}\right)$.

Proof. (1) $\Rightarrow(2)$. If $\varphi$ is a qpsh function such that $\operatorname{lct}^{\mathfrak{q}}(\varphi)=\frac{A(v)+v(\mathfrak{q})}{-\varphi(v)}$, then for any tempered valuation $w \geq v$ we have $\frac{A(v)+v(\mathfrak{q})}{-\varphi(v)} \leq \frac{A(w)+w(\mathfrak{q})}{-\varphi(w)}$ and $\varphi(w) \leq$ $\varphi(v)$. Therefore $A(w)+w(\mathfrak{q}) \geq A(v)+v(\mathfrak{q})$.

$(2) \Rightarrow(3)$. For an arbitrary tempered valuation $w$ with $\phi_{v}(w) \neq 0$, we replace $w$ by $\left(-\phi_{v}(w)\right)^{-1} w$. Therefore we can assume $\phi_{v}(w)=-1$. Since $w \geq v$ by Lemma $3.12(3)$, we have $A(w)+w(\mathfrak{q}) \geq A(v)+v(\mathfrak{q})$, and it follows that $\frac{A(w)+w(\mathfrak{q})}{-\phi_{v}(w)} \geq \frac{A(v)+v(\mathfrak{q})}{-\phi_{v}(v)}$.

$(3) \Rightarrow(1)$. Note that $\phi_{v}$ is obviously qpsh.

Definition 3.18. A nontrivial tempered valuation that satisfies one of the conditions in the previous lemma is said to be $\mathfrak{q}$-computing. Given an ideal $\mathfrak{q}$, we define

$$
\Lambda_{\mathfrak{q}}:=\left\{v \in V_{X} \mid A(v)+v(\mathfrak{q})=1\right\}
$$


and we denote the set of $\mathfrak{q}$-computing valuations in $\Lambda_{\mathfrak{q}}$ by $\Omega_{\mathfrak{q}}$. Given a qpsh function $\varphi$, the set of all valuations in $\Lambda_{\mathfrak{q}}$ which $\operatorname{compute} \operatorname{lct}^{\mathfrak{q}}(\varphi)$ is denoted by $\Omega_{\mathfrak{q}}(\varphi)$. Moreover, one defines a partial order on $\Lambda_{\mathfrak{q}}$ such that $w \succeq v$ if $\Omega_{\mathfrak{q}}\left(\phi_{w}\right) \subseteq \Omega_{\mathfrak{q}}\left(\phi_{v}\right)$.

Lemma 3.19. Let $w, v \in \Lambda_{\mathfrak{q}}$ be two tempered valuations.

(1). $v \in \Omega_{\mathfrak{q}}$ if and only if $\operatorname{lct}^{\mathfrak{q}}\left(\phi_{v}\right)=1$.

(2). $w \in \Omega_{\mathfrak{q}}\left(\phi_{v}\right)$ if and only if $w \geq\left\|\phi_{v}\right\|_{\mathfrak{q}} \cdot v$, or equivalently $\phi_{w} \geq\left(\operatorname{lct}^{\mathfrak{q}} \phi_{v}\right)$. $\phi_{v}$.

(3). If $w, v \in \Omega_{\mathfrak{q}}$. Then $w \succeq v$ if and only if $w \geq v$.

(4). If $\left\|\phi_{w}\right\|_{\mathfrak{q}} \cdot w \geq\left\|\phi_{v}\right\|_{\mathfrak{q}} \cdot v$, or equivalently $\left(\operatorname{lct}^{\mathfrak{q}} \phi_{w}\right) \cdot \phi_{w} \geq\left(\operatorname{lct}^{\mathfrak{q}} \phi_{v}\right) \cdot \phi_{v}$, then $w \succeq v$.

Proof. (1). According to Lemma 3.17(3), $v \in \Omega_{\mathfrak{q}}$ if and only if $v$ computes $\operatorname{lct}^{\mathfrak{q}} \phi_{v}$. Therefore, $\operatorname{lct}^{\mathfrak{q}} \phi_{v}=\frac{A(v)+v(\mathfrak{q})}{-\phi_{v}(v)}=1$.

(2). $w \in \Omega_{\mathfrak{q}}\left(\phi_{v}\right)$ if and only if $\operatorname{lct}^{\mathfrak{q}}\left(\phi_{v}\right)=\frac{A(w)+w(\mathfrak{q})}{-\phi_{v}(w)}$. That is, $\inf _{\mathfrak{b}} \frac{w(\mathfrak{b})}{v(\mathfrak{b})}=\left\|\phi_{v}\right\|_{\mathfrak{q}}$. This is equivalent to saying that $w \geq\left\|\phi_{v}\right\|_{\mathfrak{q}} \cdot v$.

(3). If $w, v \in \Omega_{\mathfrak{q}}$ and $w \succeq v$, then $w \in \Omega_{\mathfrak{q}}\left(\phi_{v}\right)$ by Lemma 3.17(3). Therefore $w \geq\left\|\phi_{v}\right\|_{\mathfrak{q}} \cdot v=v$ by (1). Conversely if $w \geq v$, then $\Omega_{\mathfrak{q}}\left(\phi_{w}\right)=\{u \mid u \geq w\} \subseteq$ $\{u \mid u \geq v\}=\Omega_{\mathfrak{q}}\left(\phi_{v}\right)$ by (1) and (2).

(4). If $u \in \Omega_{\mathfrak{q}}\left(\phi_{w}\right)$, then by (2) $u \geq\left\|\phi_{w}\right\|_{\mathfrak{q}} \cdot w$. It follows that $u \geq\left\|\phi_{v}\right\|_{\mathfrak{q}} \cdot v$ and by (2) again $u \in \Omega_{\mathfrak{q}}\left(\phi_{v}\right)$. We conclude that $\Omega_{\mathfrak{q}}\left(\phi_{w}\right) \subseteq \Omega_{\mathfrak{q}}\left(\phi_{v}\right)$.

Remark 3.20. On the subset $\Omega_{\mathfrak{q}}$ the partial order $\succeq$ is nothing else but $\geq$. But this is much more complicated on the whole space $\Lambda_{\mathfrak{q}}$. An interesting question is, given a tempered valuation $v \in \Lambda_{\mathfrak{q}}$, is it possible to find a valuation $w \in \Omega_{\mathfrak{q}}$ such that $\Omega_{\mathfrak{q}}\left(\phi_{v}\right)=\Omega_{\mathfrak{q}}\left(\phi_{w}\right)$ ?

Corollary 3.21. Let $v$ be a nontrivial tempered valuation, and let $\eta_{v}$ be its tameness constant. Then, we have the inequality

$$
\operatorname{lct}\left(\phi_{v}\right) \leq \eta_{v} \leq A(v) .
$$

As a result, if $v$ is computing, then $\eta_{v}=A(v)$.

Proof. The assertion follows by combining Proposition 3.6, Lemma 3.12(1) and (2).

Remark 3.22. We expect that there would be an invariant which give a criterion for a $\mathfrak{q}$-computing tempered valuation. As the previous corollary 
asserts, if $v$ is computing, then its tameness constant $\eta_{v}=A(v)$. However it is not known if the converse statement is true.

Definition 3.23. Given a nontrivial tempered valuation $v \in V_{X}^{*}$, we define the set $\Omega_{\mathfrak{q}}(v):=\left\{w \in \Lambda_{\mathfrak{q}} \mid w \geq v\right\}$.

Lemma 3.24. Let $v$ be a nontrivial tempered valuation.

(1). $\Omega_{\mathfrak{q}}(t v)$ is a closed subset of $\Lambda_{\mathfrak{q}}$ for every $t>0$, and $\Omega_{\mathfrak{q}}(t v) \supseteq \Omega_{\mathfrak{q}}\left(t^{\prime} v\right)$ if $0<t \leq t^{\prime}$.

(2). $\left\|\phi_{v}\right\|_{\mathfrak{q}}=\max \left\{t \mid \Omega_{\mathfrak{q}}(t v) \neq \emptyset\right\}$. In particular, for a valuation $v \in \Lambda_{\mathfrak{q}}, v \in$ $\Omega_{\mathfrak{q}}$ if and only if $\Omega_{\mathfrak{q}}((1+\varepsilon) v)=\emptyset$ for every $\varepsilon>0$.

Proof. (1). Note that $\Omega_{\mathfrak{q}}(t v)=\left\{w \in \Lambda_{\mathfrak{q}} \mid \phi_{t v}(w) \leq-1\right\}$ by Lemma 3.12(3). This is closed since $\phi_{t v}$ is continuous (see [[29], Section 4.2]).

$(2)$. If $\Omega_{\mathfrak{q}}(t v) \neq \emptyset$, then $\left\|\phi_{v}\right\|_{\mathfrak{q}} \geq \frac{-\phi_{v}(w)}{A(w)+w(\mathfrak{q})} \geq t$. Conversely, if there exists a tempered valuation $w \in \Lambda_{\mathfrak{q}}$ which computes $\left\|\phi_{v}\right\|_{\mathfrak{q}}$, then $w \geq\left\|\phi_{v}\right\|_{\mathfrak{q}} \cdot v$ by Lemma 3.12(3). This proves $\left\|\phi_{v}\right\|_{\mathfrak{q}} \leq \max \left\{t \mid \Omega_{\mathfrak{q}}(t v) \neq \emptyset\right\}$.

Theorem 3.25. Let $v$ be a nontrivial tempered valuation. If $r_{Y, D}(v)$ is $\mathfrak{q}$ computing for all sufficiently high log resolutions $(Y, D)$ of $X$, then $v$ is $\mathfrak{q}$-computing.

Proof. After replacing $v$ by $\frac{1}{A(v)+v(\mathfrak{q})} v$, we can assume that $v \in \Lambda_{\mathfrak{q}}$. If $v$ is not $\mathfrak{q}$-computing, then $\Omega_{\mathfrak{q}}((1+\varepsilon) v) \neq \emptyset$ for some small number $\varepsilon>0$. Therefore $\Omega_{\mathfrak{q}}\left((1+\varepsilon) r_{Y, D}(v)\right) \neq \emptyset$ since $v \geq r_{Y, D}(v)$. Assume the $\log$ resolution $(Y, D)$ is sufficiently high such that $(1+\varepsilon)\left(A\left(r_{Y, D}(v)\right)+r_{Y, D}(v)(\mathfrak{q})\right)>1+\delta$ for some sufficiently small number $\delta>0$. Let $w=\frac{1}{A\left(r_{Y, D}(v)\right)+r_{Y, D}(v)(\mathfrak{q})} r_{Y, D}(v) \in \Lambda_{\mathfrak{q}}$. Since $\Omega_{\mathfrak{q}}((1+\delta) w) \neq \emptyset$, we deduce that $w \notin \Omega_{\mathfrak{q}}$ by Lemma $3.19(1)$ and Lemma $3.24(2)$, and thus $r_{Y, D}(v)$ is not $\mathfrak{q}$-computing.

Remark 3.26. We do not know wether $\Omega_{\mathfrak{q}}$ is closed or not. The previous theorem only asserts that if $v$ lies outside $\Omega_{\mathfrak{q}}$, then $r_{Y, D}(v)$ is not $\mathfrak{q}$-computing provided that $(Y, D)$ is sufficiently high. Also, it is natural to ask if the converse statement of the previous theorem is true which is closely related to Conjecture 3.16 .

Conjecture 3.27. Let $v$ be a nontrivial tempered valuation. If $v$ is $\mathfrak{q}$ computing, then $r_{Y, D}(v)$ is $\mathfrak{q}$-computing for all sufficiently high log resolutions $(Y, D)$ of $X$. 


\section{Restrictions of valuative qpsh functions}

In this section we will discuss restrictions of (valuative) qpsh functions and their basic properties. A natural way to define the restriction of a (valuative) qpsh function is to use the fact that every qpsh function $\varphi$ is of the form $\varphi=\log \left|\mathfrak{b}_{\bullet}\right|$ where $\mathfrak{b}_{k}=\mathcal{J}(k \varphi)$ forms a subadditive sequence of ideals. Unfortunately, we will see that this definition is not a precise analogue of the restriction of qpsh function in analytic geometry via the valuative transformation (see [4]. 5.2] for valuative transform of a psh germ). One important reason that we cannot construct such an analogue is that we do not have an algebraic description of the weak topology of psh germs (see [4], Corollary $5.7])$.

Definition 4.1. Let $Z \subseteq X$ be an irreducible regular closed subscheme and let $\varphi$ be a qpsh function. If we write $\varphi_{k}=\frac{1}{k} \log |\mathcal{J}(k \varphi)|$, then we say that $\varphi$ satisfies the restriction condition to $Z$ if

(1). $\varphi_{k \mid Z}:=\frac{1}{k} \log \left|\mathcal{J}(k \varphi) \cdot \mathcal{O}_{Z}\right|$ is well-defined, that is, $\mathcal{J}(k \varphi) \cdot \mathcal{O}_{Z} \neq(0)$, for every integer $k$, and

(2). $\varphi_{k \mid Z}$ converges strongly in the norm.

In this case then we define the restriction $\varphi_{\mid Z}$ to be the limit function of $\varphi_{k \mid Z}$. Note that Condition (2) will be automatically satisfied if the qpsh function $\varphi$ is associated to a $\mathbb{Q}$-Cartier $\mathbb{Q}$-divisor $D$ or a $\mathbb{Q}$-line bundle $L$. See Proposition 5.5 .

Remark 4.2. If we work on $X=\operatorname{Spec} \widehat{R}$ where $R$ is the localization of $\mathbb{C}\left[x_{1}, \ldots, x_{n}\right]$ at the origin, then the qpsh functions are exactly the formal psh functions discussed in [4] (See [29], Remark 4.27] for an argument). If we take the analytic psh germ $\varphi=-\log \left(-\log \max \left|z_{1}\right|\right)$, then the valuative transform $\widehat{\varphi}$ is the zero function because the singularity is too mild to be detected by tempered valuations. On the other hand, the equation $\varphi(z) \equiv$ $-\infty$ holds identically on $\left\{z_{1}=0\right\}$. This example shows that the valuative transform loses some important information from complex analysis.

Lemma 4.3. (1). If $\varphi$ satisfies the restriction condition to $Z$, then so does $t \varphi$ for every $t>0$. Further, $(t \varphi)_{\mid Z}=t \varphi_{\mid Z}$.

(2). If $\varphi$ and $\psi$ satisfy the restriction condition to $Z$, then so does $\varphi+\psi$. Further, $(\varphi+\psi)_{\mid Z}=\varphi_{\mid Z}+\psi_{\mid Z}$.

Proof. (1). By definition of the multiplier ideal we have $\mathcal{J}(k t \varphi) \cdot \mathcal{O}_{Z} \neq(0)$, and by [[31], Lemma 2.6] we have that $\frac{1}{k t} \log \left|\mathcal{J}(k t \varphi) \cdot \mathcal{O}_{Z}\right|$ converges to $\varphi_{\mid Z}$ 
strongly in the norm. Therefore $t \varphi_{k \mid Z}:=\frac{1}{k} \log \left|\mathcal{J}(t k \varphi) \cdot \mathcal{O}_{Z}\right|$ form a Cauchy sequence which converges to $t \varphi_{\mid Z}$ strongly in the norm.

(2). For every integer $k$ and every sufficiently small number $\epsilon>0$, there exists a sufficiently divisible number $m$ such that $\mathcal{J}(k(\varphi+\psi))=\mathcal{J}((1+$ $\left.\epsilon) k\left(\varphi_{m}+\psi_{m}\right)\right)$ according to [[29], Lemma 4.20]. Therefore,

$$
\frac{1}{k} \log |\mathcal{J}(k(\varphi+\psi))| \geq(1+\epsilon)\left(\varphi_{m}+\psi_{m}\right)=(1+\epsilon) \frac{1}{m} \log |\mathcal{J}(m \varphi) \cdot \mathcal{J}(m \psi)|
$$

and it follows that

$$
\begin{aligned}
\left(\mathcal{J}(k(\varphi+\psi)) \cdot \mathcal{O}_{Z}\right)^{m / k(1+\epsilon)} & =\mathcal{J}(k(\varphi+\psi))^{m / k(1+\epsilon)} \cdot \mathcal{O}_{Z} \\
& \supseteq \mathcal{J}(m \varphi) \cdot \mathcal{J}(m \psi) \cdot \mathcal{O}_{Z} \\
& =\left(\mathcal{J}(m \varphi) \cdot \mathcal{O}_{Z}\right) \cdot\left(\mathcal{J}(m \psi) \cdot \mathcal{O}_{Z}\right) \neq(0) .
\end{aligned}
$$

Now we write $\Phi_{k}=\frac{1}{k} \log |\mathcal{J}(k(\varphi+\psi))|$ and $\Phi_{k \mid Z}=\frac{1}{k} \log \mid \mathcal{J}(k(\varphi+\psi))$. $\mathcal{O}_{Z} \mid$. We have

$$
\begin{aligned}
\varphi_{k \mid Z}+\psi_{k \mid Z} \geq \Phi_{k \mid Z} & \geq(1+\epsilon)\left(\varphi_{m \mid Z}+\psi_{m \mid Z}\right) \\
& \geq(1+\epsilon)\left(\varphi_{\mid Z}+\psi_{\mid Z}\right) .
\end{aligned}
$$

Since $\epsilon$ can be chosen arbitrary small, we have $\varphi_{k \mid Z}+\psi_{k \mid Z} \geq \Phi_{k \mid Z} \geq \varphi_{\mid Z}+$ $\psi_{\mid Z}$. Thus $\Phi_{k \mid Z}$ converges to $\varphi_{\mid Z}+\psi_{\mid Z}$ strongly in the norm.

The previous lemma shows that qpsh functions which satisfy the restriction condition to $Z$ form a convex subcone of $\operatorname{QPSH}(X)$. We denote this subcone by $\operatorname{QPSH}_{Z}(X)$. Like [[29], Lemma 4.16, Proposition 6.10 and Proposition 6.11], it is natural to ask the following question.

Question 4.4. Is the cone $\mathrm{QPSH}_{Z}(X)$ closed under taking the supremum?

We will only give the affirmative answer in some cases (see Proposition 5.9p. However, we expect this to hold unconditionally.

Remark 4.5. Note that the cone $\operatorname{QPSH}_{Z}(X)$ is NOT closed in norm. We will give a counter example later (See Example 5.11).

Theorem 4.6 (Restriction Theorem I). If $\varphi$ is a qpsh function which satisfies the restriction condition to $Z$, then we have the inclusion

$$
\mathcal{J}\left(\varphi_{\mid Z}\right) \subseteq \mathcal{J}(\varphi) \cdot \mathcal{O}_{Z}
$$


Proof. By the definition of the restriction condition, $\varphi_{\mid Z}$ is approximated by $\varphi_{k \mid Z}$ strongly in the norm. Therefore from [[29], Lemma 4.20] there exists a small number $\epsilon>0$ such that $\mathcal{J}\left(\varphi_{\mid Z}\right)=\mathcal{J}\left((1+\epsilon) \varphi_{k \mid Z}\right)$ and $\mathcal{J}(\varphi)=\mathcal{J}((1+$ $\epsilon) \varphi_{k}$ ) for every sufficiently divisible integer $k$. Now we apply [[31], Theorem A.1] and hence conclude this inclusion.

Definition 4.7 (Local log canonical threshold). Let $X$ be a scheme and $\xi$ be a point of $X$. The inclusion $\operatorname{SpecO}_{X, \xi} \rightarrow X$ induces an inclusion of tempered valuation spaces $V_{\mathrm{Spec}} \mathcal{O}_{X, \xi} \rightarrow V_{X}$ which preserves the log discrepancy. If $\varphi$ is a qpsh function on $X$, then we define $\varphi_{\xi}$ to be the pull-back of $\varphi$. In this case, we denote the log canonical threshold of $\varphi_{\xi}$ by $\operatorname{lct}_{\xi}(\varphi)$. It is easy to see that $\operatorname{lct}_{\xi}(\varphi) \leq \operatorname{lct}_{\xi^{\prime}}(\varphi)$ if $\xi$ dominates $\xi^{\prime}$. Further, if $Z$ is a closed subset of $X$, then we define the $\log$ canonical threshold of $\varphi$ on $Z$ to be $\operatorname{lct}_{Z}(\varphi):=\inf _{\xi \in Z} \operatorname{lct}_{\xi}(\varphi)$. Clearly, if $Z^{\prime} \subseteq Z$, then $\operatorname{lct}_{Z}(\varphi) \leq \operatorname{lct}_{Z^{\prime}}(\varphi)$.

It is easy to check that, if a qpsh function $\varphi$ is induced by a finite number of ideals $\mathfrak{a}_{i}$ in the sense of $\varphi=\sum c_{i} \log \left|\mathfrak{a}_{i}\right|$, then $\operatorname{lct}_{\xi}(\varphi)$ is precisely the $\log$ canonical threshold of $\prod \mathfrak{a}_{i}^{c_{i}}$ at $\xi$. Similarly if $\varphi$ is induced by a graded sequence of ideals $\mathfrak{a}_{\bullet}$, then $\operatorname{lct}_{\xi}(\varphi)$ is exactly the local $\log$ canonical threshold $\operatorname{lct}_{\xi}\left(\mathfrak{a}_{\bullet}\right)$. For details, we refer to [29], Section 3.2].

Theorem 4.8 (Restriction Theorem II). Let $\varphi$ be a qpsh function which satisfies the restriction condition to $Z$. Given a point $\xi \in Z$, we have the inequality of norms $\left\|\varphi_{\mid Z, \xi}\right\| \geq\left\|\varphi_{\xi}\right\|$, or equivalently of log canonical thresholds, $\operatorname{lct}_{\xi}\left(\varphi_{\mid Z}\right) \leq \operatorname{lct}_{\xi}(\varphi)$. In particular, given a closed subset $Y$ of $Z$, we have $\operatorname{lct}_{Y}\left(\varphi_{\mid Z}\right) \leq \operatorname{lct}_{Y}(\varphi)$.

Proof. After replacing $X$ and $Z$ by $\operatorname{Spec} \mathcal{O}_{X, \xi}$ and $\operatorname{Spec} \mathcal{O}_{Z, \xi}$ respectively, we will show that $\operatorname{lct}\left(\varphi_{\mid Z}\right) \leq \operatorname{lct}(\varphi)$. If we set $\lambda:=\operatorname{lct}\left(\varphi_{\mid Z}\right)$, then we have $\mathcal{J}\left((\lambda-\epsilon) \varphi_{\mid Z}\right)=\mathcal{O}_{Z}$ for every $\epsilon>0$. By theorem 4.6 we have $\mathcal{O}_{Z}=\mathcal{J}((\lambda-$ $\left.\epsilon) \varphi_{\mid Z}\right) \subseteq \mathcal{J}((\lambda-\epsilon) \varphi) \cdot \mathcal{O}_{Z}$. Therefore $\mathcal{J}((\lambda-\epsilon) \varphi)$ contains the maximal ideal $\mathfrak{m}_{\xi}$ properly since $\mathfrak{m}_{Z} \subseteq \mathfrak{m}_{\xi}$, which implies that $\mathcal{J}((\lambda-\epsilon) \varphi)=\mathcal{O}_{X}$ and hence $\lambda \leq \operatorname{lct}(\varphi)$. The last assertion follows from the definition directly.

Remark 4.9. The readers could compare the previous result to [[15], 2.2. Proposition]. Note that if an analytic psh function $\varphi$ has algebraic singularities, then the complex singularity exponents $c_{K}(\varphi)$ on an irreducible analytic set $K$ is exactly the log canonical threshold of its corresponding "algebraic data" on $K$. As we mentioned in Remark 4.2, the restriction of a valuative qpsh function is not a precise analogue of that in complex analysis. For instance, if we take an analytic psh function $\varphi$ and denote its valuative 
transform by $\widehat{\varphi}$, then we have $c_{K}(\varphi)=\operatorname{lct}_{K}(\widehat{\varphi})$ by [[15], $\left.3.2 \operatorname{Theorem}(3)\right]$. But in general, $c_{K}\left(\varphi_{\mid Z}\right)$ can be very different from $\operatorname{lct}_{K}\left(\widehat{\varphi}_{\mid Z}\right)$.

Proposition 4.10. Let $Z \subseteq X$ be a regular closed irreducible subscheme. If $\varphi$ is a tame qpsh function, then $\varphi$ satisfies the restriction condition to $Z$ if and only if $\varphi\left(\operatorname{ord}_{\xi}\right)=0$ where $\xi$ is the generic point of $Z$. In this case, $\mathfrak{a}(k \varphi)$. $\mathcal{O}_{Z} \neq(0)$ for every integer $k>0$ and $\varphi_{\mid Z}$, which is of the form $\log |\mathfrak{a}(\varphi) \bullet| Z \mid$, is also tame.

Proof. By definition there exists a constant $C>0$ such that $\log \mid \mathcal{J}((C+$ t) $\varphi) \mid \leq t \varphi$. It follows that $\varphi\left(\operatorname{ord}_{\xi}\right) \leq \operatorname{ord}_{\xi} \mathcal{J}((C+1) \varphi)=0$ provided that $\varphi$ satisfies the restriction condition to $Z$. Conversely if $\varphi\left(\operatorname{ord}_{\xi}\right)=0$, then we have $\mathfrak{a}(k \varphi) \cdot \mathcal{O}_{Z} \supseteq \mathcal{J}((k+C) \varphi) \cdot \mathcal{O}_{Z} \neq(0)$. Set $\psi_{k}=\frac{1}{k} \log |\mathfrak{a}(k \varphi)|$ and $\psi_{k \mid Z}=\frac{1}{k} \log \left|\mathfrak{a}(k \varphi) \cdot \mathcal{O}_{Z}\right|$, and hence $\varphi_{k \mid Z} \geq \psi_{k \mid Z} \geq\left(1+\frac{C}{k}\right) \varphi_{k \mid Z}$. Since $\mathfrak{a}_{\bullet}, Z:=\left\{\mathfrak{a}(k \varphi) \cdot \mathcal{O}_{Z}\right\}_{k=1}^{\infty}$ is a graded sequence of ideals, $\psi_{k \mid Z}$ converges strongly in the norm. We obtain that $\varphi_{k \mid Z}$ converges to $\varphi_{\mid Z}$ strongly in the norm.

To see that $\varphi_{\mid Z}$ is tame, it suffices to consider the inclusions

$$
\mathcal{J}\left((k+C) \varphi_{\mid Z}\right) \subseteq \mathcal{J}((k+C) \varphi) \cdot \mathcal{O}_{Z} \subseteq \mathfrak{a}(k \varphi) \cdot \mathcal{O}_{Z} \subseteq \mathfrak{a}\left(k \varphi_{\mid Z}\right)
$$

as an application of Theorem 4.6 .

Corollary 4.11. If $\varphi=\sum_{i=1}^{k} c_{i} \log \left|\mathfrak{a}_{i}\right|$ is an ideal function, then $\varphi$ satisfies the restriction condition to $Z$ if and only if all $\mathfrak{a}_{i}$ do not vanish along $Z$. In this case, $\varphi_{\mid Z}=\sum_{i=1}^{k} c_{i} \log \left|\mathfrak{a}_{i} \cdot \mathcal{O}_{Z}\right|$.

Proof. This assertion follows by combining Proposition 4.10, Corollary 3.9 and Lemma 4.3 .

Inversion of adjunction. The aim of this subsection is to prove the following theorem which is a version of inversion of adjunction. In the theory of classification of higher dimensional varieties inversion of adjunction provides a central tool. This was originally proved by V. Shokurov in [43] in dimension 3 and generalized to all dimensions by J. Kollár in [34]. Here is a non-exhaustive list of references in recent progress: [28], 33], 44], [45], [18], [19], 2].

Theorem 4.12. Let $H \subseteq X$ be an irreducible regular closed subscheme of codimension one, and let $\varphi$ be a qpsh function which satisfies the restriction 
condition to $H$. Write $\psi=\log \left|\mathcal{O}_{X}(-H)\right|$. Given a point $\xi \in H, \operatorname{lct}_{\xi}\left(\varphi_{\mid H}\right) \geq$ 1 if and only if $\operatorname{lct}_{\xi}(\varphi+\psi) \geq 1$. In particular, given a closed subset $Z$ of $H$, $\operatorname{lct}_{Z}\left(\varphi_{\mid H}\right) \geq 1$ if and only if $\operatorname{lct}_{Z}(\varphi+\psi) \geq 1$.

Proof. Note that the assertion is equivalent to saying that $\left\|\varphi_{\mid H, \xi}\right\| \leq 1$ if and only if $\left\|\varphi_{\xi}+\psi_{\xi}\right\| \leq 1$. According to [[31], Proposition 1.9] and [[29], Proposition 4.22], we can replace $X$ by $\operatorname{Spec} \widehat{\mathcal{O}_{X, \xi}}$ (See [[31], Appendix A] for more details). Therefore, by Cohen's structure theorem we can assume that $X=\operatorname{Spec} k\left[\left[x_{1}, \ldots, x_{m}\right]\right]$, for some $m$, and that $H$ is defined by the ideal $\left(x_{1}\right)$. In particular, we can assume that $\xi$ is a closed point of $X$.

Now we prove the "only if" part since the "if" part is easier and can be argued similarly. Assume that $\operatorname{lct}_{\xi}\left(\varphi_{\mid H}\right) \geq 1$. It follows that $\mathcal{J}\left((1-\epsilon) \varphi_{k \mid H}\right)_{\xi}$ is trivial for every sufficiently large integer $k$ and every sufficiently small number $\epsilon>0$ by [[29], Lemma 4.20]. Recall that $\varphi_{k}=\frac{1}{k} \log |\mathcal{J}(k \varphi)|$ as we denoted earlier. From the fact that $\mathcal{J}\left((1-\epsilon) \varphi_{k \mid H}\right)=\bigcap_{N} \mathcal{J}\left(\left(\mathcal{J}(k \varphi)_{H}+\right.\right.$ $\left.\left.\mathfrak{m}_{H}^{N}\right)^{\frac{1-\epsilon}{k}}\right)$ and $\mathcal{J}\left((1-\epsilon)\left(\varphi_{k}+\psi\right)\right)=\bigcap_{N} \mathcal{J}\left(\left(\mathcal{J}(k \varphi) \cdot \mathcal{O}_{X}(-H)^{k}+\mathfrak{m}^{N}\right)^{\frac{1-\epsilon}{k}}\right)$, where $\mathcal{J}(k \varphi)_{H}$ and $\mathfrak{m}_{H}$ denotes the restriction of $\mathcal{J}(k \varphi)$ and $\mathfrak{m}$ to $H$ respectively, we only need to show that $\mathcal{J}\left(\left(\mathcal{J}(k \varphi) \cdot \mathcal{O}_{X}(-H)^{k}+\mathfrak{m}^{N}\right)^{\frac{1-\epsilon}{k}}\right)$ is trivial for every $N$. For this reason we can assume that $X=\mathbb{A}_{k}^{m}$. By [[31], Example 1.1] we can assume that $k$ is algebraically closed.

Pick a general member $f_{k}$ of the ideal $\mathcal{J}(k \varphi)$ and denote the corresponding divisor by $D_{k}$. It is obvious that $f_{k \mid H}$ is also a general member of $\mathcal{J}(k \varphi)_{H}$. Therefore, by usual inversion of adjunction (for example, see [[19], Theorem 0.1]) and [[36], Proposition 9.2.28], we obtain that $\mathcal{J}\left((1-\epsilon)\left(\varphi_{k}+\psi\right)\right)=$ $\left.\mathcal{J}\left(\frac{1-\epsilon}{k} D_{k}+(1-\epsilon) H\right)\right)$ is trivial from that $\mathcal{J}\left((1-\epsilon) \varphi_{k \mid H}\right)=\mathcal{J}\left(\frac{1-\epsilon}{k} D_{k \mid H}\right)$ is trivial, and hence $\operatorname{lct}_{\xi}(\varphi+\psi) \geq 1$.

Remark 4.13. The readers could compare the previous theorem with [[15], 2.5. Theorem].

\section{Applications}

If $X$ is a smooth complex projective variety, then we can associate a valuative qpsh function to a line bundle. This has been studied for ideal functions (see Definition 2.4) in many relevant references such as [3], [16], [17, [20], [37, [40]. We developed such a theory for qpsh functions in [29], Section 6]. Besides, it might be possible to generalize the results to varieties with mild singularities such as klt singularities (see [8], [9]). 
Throughout this section $X$ will be a projective smooth variety over $\mathbb{C}$ for simplicity. The term "divisor" will always refer to a $\mathbb{Q}$-Cartier $\mathbb{Q}$-divisor. Given a section $s \in H^{0}(X, L)$ of a line bundle, the notation $\log |s|$ denotes the qpsh function defined locally by a regular function corresponding to $s$.

Restriction of D-psh functions. To begin with, we briefly review the definitions and propositions from [29], Section 6].

Definition 5.1. Given a divisor $D$, we define the set

$$
\begin{aligned}
\mathcal{L}_{D}:= & \left\{\frac{1}{k} \log |\mathfrak{a}| \mid k m D \otimes \mathfrak{a}^{m}\right. \text { is globally generated } \\
& \text { for every sufficiently divisible } m\} .
\end{aligned}
$$

We then define the set of $D$-psh functions to be the closure $\operatorname{PSH}(D)=\overline{\mathcal{L}_{D}}$ in the norm.

Definition 5.2. The set of pseudo D-psh functions is defined to be $\operatorname{PSH}_{\sigma}(D):=\bigcap_{\varepsilon>0} \operatorname{PSH}(D+\varepsilon A)$ where $A$ is an ample divisor.

Note that the above definition is independent of the choice of the ample divisor $A$. As in complex algebraic geometry, we have the corresponding vanishing theorem, global generation theorem, etc. We will frequently use them and we present here for the reader's convenience. See [[29], Theorem 6.5, Theorem 6.6] for proofs and more details.

Theorem 5.3 (Nadel Vanishing). Let $L$ be a line bundle on a smooth projective variety $X$ and $L \equiv A+D$ where $A$ is a nef and big $\mathbb{Q}$-divisor. Assume that $\varphi \in \operatorname{PSH}_{\sigma}(D)$. Then

$$
H^{i}\left(X,\left(K_{X}+L\right) \otimes \mathcal{J}(\varphi)\right)=0
$$

for all $i>0$.

Theorem 5.4 (Global generation). Let $D$ be a divisor on $X$ and $\varphi$ be a qpsh function. Then, $\varphi$ is pseudo D-psh if and only if there exists a line bundle $G$ such that $(m D+G) \otimes \mathcal{J}(m \varphi)$ is globally generated for all $m \in \mathbb{Z}_{+}$ with $m D$ integral.

Now we discuss of the restriction of a pseudo $D$-psh function and that of a $D$-psh function. An important feature of a pseudo $D$-psh function is that condition (2) in Definition 4.1 automatically holds as long as condition (1) is satisfied. 
Proposition 5.5. Let $Z \subseteq X$ be a smooth closed subvariety, let $D$ be a divisor and let $\varphi$ be a pseudo D-psh function. If we write $\varphi_{k}=\frac{1}{k} \log |\mathcal{J}(k \varphi)|$, then $\varphi$ satisfies the restriction condition to $Z$ if $\varphi_{k \mid Z}:=\frac{1}{k} \log \left|\mathcal{J}(k \varphi) \cdot \mathcal{O}_{Z}\right|$ is well-defined, that is, $\mathcal{J}(k \varphi) \cdot \mathcal{O}_{Z} \neq(0)$, for every integer $k$, or equivalently $\varphi\left(\operatorname{ord}_{Z}\right)=0$.

Before we prove this proposition, we show the following useful lemmas as preparation.

Lemma 5.6. Let $D$ be a divisor on $X$, and let $\left\{\phi_{k}\right\}$ be a decreasing sequence of pseudo D-psh functions. Then, $\phi_{k}$ converges to a pseudo D-psh function strongly in the norm.

Proof. For every integer $m$, the sequence of ideals $\left\{\mathcal{J}\left(m \phi_{k}\right)\right\}$ satisfies descending chain condition as a result of Theorem 5.4. That is, $\mathcal{J}\left(m \phi_{k}\right)$ stabilizes when $k \geq N(m)$, which in turn implies that $\left\|\phi_{k}-\phi_{k^{\prime}}\right\|<\frac{1}{m}$ for $k$ and $k^{\prime} \geq N(m)$.

Now we can prove the following stronger version of Proposition 5.5

Corollary 5.7. Let D, $Z, X$ and $\varphi$ be as in Proposition 5.5. Then, the function $\varphi_{\mid Z}$ is well-defined and pseudo $\left.D\right|_{Z}$-psh.

Proof. There exists an ample divisor $A$ such that $\varphi_{k} \in \mathcal{L}_{D+A}$ for every integer $k$ by Theorem 5.4. It follows that $\varphi_{k \mid Z} \in \mathcal{L}_{\left.D\right|_{z}+\left.A\right|_{z}}$ for all $k$. Note that $\left\{\mathcal{J}(k \varphi) \cdot \mathcal{O}_{Z}\right\}$ is a subadditive sequence of ideals. Therefore, $\left\{\varphi_{k \mid Z}\right\}$ has a decreasing subsequence of $\left.D\right|_{Z}+\left.A\right|_{Z}$-psh functions (see [29], Remark 4.25]) which converges to the limit function of $\varphi_{k \mid Z}$ strongly in the norm by [[31], Lemma 2.6] and Lemma 5.6

In the previous corollary we see that being pseudo $D$-psh can be "preserved" under restricting to a smooth closed subvariety. However, if we assume that $\varphi$ is $D$-psh, then $\varphi_{\mid Z}$ is not necessarily $\left.D\right|_{Z}$-psh.

Example 5.8. Let $D$ be a nef and abundant divisor on $X$, and let $Z$ be a smooth hypersurface of a variety $X$ such that $\left.D\right|_{Z}$ is not abundant. It is easy to see that the zero function $\phi$ is $D$-psh and its restriction $\left.\phi\right|_{Z}$ is also a zero function. But $\left.\phi\right|_{Z}$ is not $\left.D\right|_{Z}$-psh (see [29], Corollary 5.13(2)]).

Proposition 5.9. Let $D, Z$ and $X$ be as in Proposition 5.5, and let $\left\{\phi_{k}\right\}$ be a decreasing sequence of pseudo D-psh functions such that $\phi_{k}$ satisfies the 
restriction condition to $Z$ for every integer $k$. If we denote the limit function of $\phi_{k}$ by $\varphi$, then $\varphi_{\mid Z}$ is well-defined and $\lim _{k \rightarrow \infty} \phi_{k \mid Z}=\varphi_{\mid Z}$.

Proof. Since $\mathcal{J}(m \varphi)=\mathcal{J}\left((1+\epsilon) m \phi_{k}\right)$ for a sufficiently small number $\epsilon$ and every sufficiently divisible integer $k$ by [[29], Lemma 4.20], we have $\varphi_{m} \geq$ $(1+\epsilon) \phi_{k}$ and hence $\varphi_{m \mid Z}$ is well-defined such that $\varphi_{m \mid Z} \geq(1+\epsilon) \phi_{k \mid Z}$. By Lemma 5.6 we have that $\phi_{k \mid Z}$ converges to a function. Now we obtain that $\varphi_{m \mid Z} \geq \lim _{k \rightarrow \infty}(1+\epsilon) \phi_{k \mid Z}$ and hence $\varphi_{m \mid Z} \geq \lim _{k \rightarrow \infty} \phi_{k \mid Z}$ since $\epsilon$ can be chosen arbitrary small. By Proposition 5.5. $\varphi_{\mid Z}=\lim _{m \rightarrow \infty} \varphi_{m \mid Z}$ is well-defined and $\lim _{k \rightarrow \infty} \phi_{k \mid Z} \leq \varphi_{\mid Z}$. On the other hand, we have $\lim _{k \rightarrow \infty}^{\infty} \phi_{k \mid Z} \geq \varphi_{\mid Z}$ since $\phi_{k} \geq \varphi$ for every integer $k$. Therefore we obtain the equality.

Remark 5.10. The previous proposition asserts that the definition of the restriction of a pseudo $D$-psh function $\varphi$ is independent of the choice of a decreasing sequence of ideal functions which converges to $\varphi$ although the multiplier ideals provide us a natural way. As an application of the previous discussions we construct an example below to give a negative answer of [[29], Question 6.3].

Example 5.11. Let $X=\mathbb{C P}^{2}, U \cong \mathbb{C}^{2} \subseteq X, Z$ be the closure of the $z_{2}$-axis, and let $\phi_{k}=\sum_{l=1}^{k} \frac{1}{2^{l}} \log \left|f_{l}\right|$ where $f_{l}=z_{1}+z_{2}^{2^{l}}$ on $U$. Note that $\phi_{k}$ converges to a qpsh function $\varphi$ strongly in the norm because $\left\|\phi_{m}-\phi_{n}\right\| \leq \frac{1}{2^{m}}$ when $m<n$. We can explicitly compute that $\phi_{k \mid Z}=k \log \left|\mathfrak{m}_{0}\right|$ by Lemma 4.3(2), where $\mathfrak{m}_{0}=\left\{z_{2}=0\right\}$ on $Z$. Therefore $\phi_{k \mid Z}$ doe not converge and $\phi_{\mid Z}$ is not well-defined. Hence, $\varphi$ is not pseudo $D$-psh for any divisor $D$ by Proposition 5.5 and Proposition 5.9.

Proposition 5.12. Let $D, Z$ and $X$ be as in Proposition 5.5, and let $\phi$ be a set of pseudo D-psh functions such that $\phi_{\lambda}$ satisfies the restriction condition to $Z$ for every index $\lambda$. Then, $\sup _{\lambda} \varphi_{\lambda}$ satisfies the restriction condition to $Z$ and $\left.\left(\sup _{\lambda} \varphi_{\lambda}\right)\right|_{Z}=\sup _{\lambda} \varphi_{\lambda \mid Z}$. 
Proof. The first assertion follows directly from Proposition 5.5. To see the second assertion, we notice that

$$
\begin{aligned}
\left.\left(\sup _{\lambda} \varphi_{\lambda}\right)\right|_{Z} & =\lim _{k \rightarrow \infty}\left(\sup _{\lambda} \varphi_{\lambda}\right)_{k \mid Z} \\
& =\lim _{k \rightarrow \infty} \sup _{\lambda} \varphi_{\lambda, k \mid Z} \\
& \geq \sup _{\lambda} \lim _{k \rightarrow \infty} \varphi_{\lambda, k \mid Z} \\
& =\sup _{\lambda} \varphi_{\lambda \mid Z}
\end{aligned}
$$

where the second equality follows from the definition of multiplier ideals and Proposition 5.9.

Now we prove that the inequality appeared above is in fact equality. Because $\sup _{\lambda} \varphi_{\lambda \mid Z}$ can be approximated by $\sup _{\lambda} \varphi_{\lambda \mid Z, k}$, we can replace $\Lambda$ by a countable subset. We consider the sequences $\left\{\varphi_{\lambda, t \mid Z}\right\}_{t \in \mathbb{R}_{\geq 0}}$. Given a nontrivial tempered valuation $v$ and an arbitrary small number $\epsilon>0$, there exists some $\lambda_{0}$ such that $\lim _{t \rightarrow \infty} \sup _{\lambda} \varphi_{\lambda, t \mid Z}(v)-\epsilon<\lim _{t \rightarrow \infty} \varphi_{\lambda_{0}, t \mid Z}(v)$. Hence $\lim _{k \rightarrow \infty} \sup _{\lambda} \varphi_{\lambda, k \mid Z} \leq \sup _{\lambda} \lim _{k \rightarrow \infty}{\stackrel{t \rightarrow \infty}{\varphi_{\lambda, k \mid Z}}}$ and we obtain the conclusion.

Extensions of pluri-canonical forms on a dlt pair. Now we discuss the extension problem of pluri-canonical forms as an application of the above constructions. Recall that the augmented base locus of a big line bundle $L$ is defined to be $\mathbf{B}_{+}(L)=\bigcap_{A} \mathbf{B}(L-A)$ where $A$ runs over all ample divisors, and that the restricted base locus of a pseudo-effective line bundle $L$ is defined to be $\mathbf{B}_{-}(L)=\bigcup_{A} \mathbf{B}(L+A)$ where $A$ runs over all ample divisors. We use the notations $v\left(\|L\|_{Z}\right):=v\left(\mathfrak{a}_{\bullet} \mid Z\right)$ where $\mathfrak{a}_{k \mid Z}=\mathfrak{b}(\lfloor k L\rfloor \mid) \cdot \mathcal{O}_{Z}$ and $\sigma_{v}\left(\|L\|_{Z}\right):=\sup _{A} v\left(\|L+A\|_{Z}\right)$ where $A$ runs over all ample divisors.

Lemma 5.13. Let $Z$ be a smooth closed subvariety, let $L$ be a big line bundle on $X$ such that the augmented base locus $\mathbf{B}_{+}(L)$ does not contain $Z$, and let $\varphi$ be the maximal $L$-psh function, or explicitly, $\varphi(v)=-v(\|L\|)$ (see [[29], Proposition 6.10]). Then, $\varphi_{\mid Z}$ is well-defined and $\varphi_{\mid Z}(v)=-v\left(\|L\|_{Z}\right)$.

Proof. Note that $\varphi$ is weakly tame (see Example 3.4). In particular, by the proof of [[36], Theorem 11.2.21] there exists a divisor $E \geq 0$ such that $E$ does not contain $Z$ in its support and

$$
\mathcal{J}(m \varphi) \cdot \mathcal{O}_{X}(-E) \subseteq \mathfrak{b}(|m L|)
$$


for every integer $m>0$. It follows that

$$
\mathcal{J}(m \varphi) \cdot \mathcal{O}_{Z}\left(-\left.E\right|_{Z}\right) \subseteq \mathfrak{b}(|m L|) \cdot \mathcal{O}_{Z}
$$

for every sufficiently divisible integer $m>0$. Therefore, $\left.\varphi\right|_{\mid Z}=\log \left|\mathfrak{a}_{\bullet}\right| Z \mid$ where $\mathfrak{a}_{k \mid Z}=\mathfrak{b}(|\lfloor k L\rfloor|) \cdot \mathcal{O}_{Z}$.

Lemma 5.14. Let $Z$ be a smooth closed subvariety, let $L$ be a pseudoeffective line bundle on $X$ such that the restricted base locus $\mathbf{B}_{-}(L)$ does not contain $Z$, and let $\varphi$ be the maximal pseudo L-psh function, or explicitly, $\varphi(v)=-\sigma_{v}(\|L\|)$ (see [[29], Proposition 6.11]). Then, $\varphi_{\mid Z}$ is well-defined and $\varphi_{\mid Z}(v)=-\sigma_{v}\left(\|L\|_{Z}\right)$.

Proof. Fix an ample divisor $A$, and we have that $\varphi=\lim _{k \rightarrow \infty} \phi_{k}$ where $\phi_{k}$ is the maximal $\left(L+\frac{1}{k} A\right)$-psh function. Because $\mathbf{B}_{+}\left(L+\frac{1}{k} A\right)$ does not contain $Z, \phi_{k \mid Z}$ is well-defined and $\phi_{k \mid Z}(v)=-v\left(\left\|L+\frac{1}{k} A\right\|_{Z}\right)$ by Lemma 5.13 . Therefore by Proposition 5.9 we have $\varphi_{\mid Z}=\lim _{k \rightarrow \infty} \phi_{k \mid Z}$ which gives the conclusion.

The notion of adjoint ideals is crucial in birational algebraic geometry. It is powerful in inductive proofs with the aid of Nadel vanishing. Here we introduce the adjoint ideal of a pseudo $D$-psh function along a reduced simple normal crossing (snc for short) divisor.

Definition 5.15. Let $S=\sum_{i=1}^{l} S_{i}$ be a reduced divisor with its support snc on $X$, and let $\varphi$ be a pseudo $D$-psh function such that $\varphi_{\mid Z}$ is well-defined

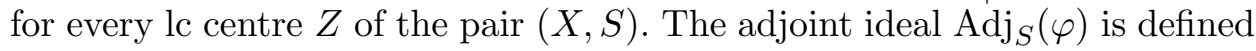
to be

$$
\operatorname{Adj}_{S}\left((1+\epsilon) \varphi_{k}\right):=\operatorname{Adj}_{S}\left(\mathcal{J}(k \varphi)^{\frac{1+\epsilon}{k}}\right)
$$

for a sufficiently small number $\epsilon>0$ and every sufficiently divisible integer $k>0$.

Lemma 5.16. The adjoint ideal appeared in the above definition is welldefined. Further, if there is a decreasing sequence of $(D+A)$-psh functions $\phi_{k}$ such that $\varphi=\lim _{k \rightarrow \infty} \phi_{k}$ for an ample divisor $A$, then $\operatorname{Adj}_{S}(\varphi)=\operatorname{Adj}_{S}((1+$ $\left.\epsilon) \phi_{m}\right)$ for a sufficiently small number $\epsilon>0$ and every sufficiently divisible integer $m>0$.

Proof. We only prove the latter assertion by induction on the number of components of $S$ which essentially implies the first statement. If $l=1$, then 
$S$ is a smooth hypersurface and hence we have the following exact sequence of ideals

$$
0 \longrightarrow \mathcal{J}(\varphi) \cdot \mathcal{O}_{X}(-S) \longrightarrow \operatorname{Adj}_{S}\left((1+\epsilon) \phi_{k}\right) \longrightarrow \mathcal{J}\left(\varphi_{\mid S}\right) \longrightarrow 0
$$

for a sufficiently small number $\epsilon>0$ and every sufficiently divisible integer $k>0$ by definition. It is easy to check that $\operatorname{Adj}_{S}\left((1+\epsilon) \varphi_{k}\right)$ stabilizes when is $\epsilon$ sufficiently small and $k$ is sufficiently divisible by 5 -lemma. Now we assume that the adjoint ideal is well-defined for $l-1$. If we write $S=S^{\prime}+S_{l}$ where $S_{l}$ is an irreducible component, then we have the following exact sequence

$$
0 \longrightarrow \operatorname{Adj}_{S^{\prime}}(\varphi) \cdot \mathcal{O}_{X}\left(-S_{l}\right) \longrightarrow \operatorname{Adj}_{S}\left((1+\epsilon) \phi_{k}\right) \longrightarrow \operatorname{Adj}_{\left.S^{\prime}\right|_{S_{l}}}\left(\varphi_{\mid S_{l}}\right) \longrightarrow 0
$$

for a sufficiently small number $\epsilon>0$ and every sufficiently divisible integer $k>0$ by Proposition 5.9 and the inductive assumption which implies the conclusion.

Remark 5.17. The previous lemma shows that the definition of adjoint ideal is independent of the choice of a decreasing sequence of ideal functions which converges to $\varphi$.

We apply the above constructions to prove the following extension of pluri-canonical forms on a dlt pair. See [[14], Theorem 1.7] and [[23], Proposition 5.11] for a comparison.

Let $(X, S+B)$ be a dlt pair such that $\lfloor S+B\rfloor=S$. Assume that the restricted base locus $\mathbf{B}_{-}\left(K_{X}+S+B\right)$ does not contain any lc centre of $(X, S+B)$. Let $\pi: X^{\prime} \rightarrow X$ be a $\log$ resolution of $(X, S+B)$ and we write

$$
K_{X^{\prime}}+S^{\prime}+B^{\prime}=\pi^{*}\left(K_{X}+S+B\right)+E^{\prime}
$$

where $\left(X^{\prime}, S^{\prime}+B^{\prime}\right)$ is dlt such that $S^{\prime}+B^{\prime}$ and $E^{\prime} \geq 0$ does not have common components and $\left\lfloor S^{\prime}+B^{\prime}\right\rfloor=S^{\prime}$. Following [30, [42, [14] and [10], if we consider the extension obstruction divisor

$$
\Xi:=\left.N_{\sigma}\left(\left\|K_{X^{\prime}}+S^{\prime}+B^{\prime}\right\|_{S^{\prime}}\right) \wedge B^{\prime}\right|_{S^{\prime}}
$$

on $S^{\prime}$ for every $\log$ resolution $X^{\prime}$, then we have the following result.

Proposition 5.18. Let $(X, S+B)$ be a dlt pair such that $\lfloor S+B\rfloor=S$. Assume that

(1). there exists an effective divisor $D \sim_{\mathbb{Q}} K_{X}+S+B$ such that $D$ contains $S$ in its support, 
(2). the restricted base locus $\mathbf{B}_{-}\left(K_{X}+S+B\right)$ does not contain any lc centre, and

(3). $K_{X}+S+B$ is abundant.

Let $m$ be an integer such that $m\left(K_{X}+S+B\right)$ is Cartier. If $\sigma$ is a section of $m\left(K_{S}+B_{S}\right)$ such that for every log resolution of $(X, S+B)$ we have

$$
\operatorname{div} \pi^{*}(\sigma)+\left.m E^{\prime}\right|_{S} \geq m \Xi
$$

then $\sigma$ extends to $X$.

Proof. After replacing $(X, S+B)$ by a log resolution, we can assume that $(X, S+B)$ is $\log$ smooth. We divide the proof into three steps.

Step 1. Let $\varphi$ be the maximal $\left(K_{X}+S+B\right)$-psh function. If we write $S=\sum_{i=1}^{l} S_{i}$, then we will show that $\log \left|\sigma_{\left|S_{i}\right|}\right| \leq m \varphi_{\mid S_{i}}$ for every $i$. To this end, we choose an ample divisor $A$ and fix an index $i$. For every sufficiently large integer $q$, we have $K_{X}+S+B+\frac{1}{q} A \sim_{\mathbb{Q}} K_{X}+S_{i}+A_{q}+\Delta_{q}$ such that

- $\left(X, S_{i}+A_{q}+\Delta_{q}\right)$ is plt and $A_{q}$ is ample;

- $\operatorname{Supp}\left(\Delta_{q}\right) \subseteq \operatorname{Supp}\left(S-S_{i}+B\right)$.

If we write $K_{X^{\prime}}+S_{i}^{\prime}+A_{q}^{\prime}+\Delta_{q}^{\prime}=\pi^{*}\left(K_{X}+S_{i}+A_{q}+\Delta_{q}\right)+E_{q}^{\prime}$ such that $S_{i}^{\prime}$ is the birational transformation of $S_{i}, A_{q}^{\prime}$ is ample, the effective divisors $\Delta_{q}^{\prime}$ and $E_{q}^{\prime}$ have no common components and the pair $\left(S_{i}, A_{q \mid S_{i}}+\Delta_{q \mid S_{i}}\right)$ has terminal singularities. If $s_{A}$ is a section of $\mathcal{O}_{X}(A)$, then for a sufficiently divisible integer $l$ we have

$$
\operatorname{div} \pi^{*}\left(\sigma_{\mid S_{i}}^{l} \cdot s_{A \mid S_{i}}^{l m / q}\right)+\operatorname{lm} E_{q}^{\prime} \geq \operatorname{lm} \Delta_{q \mid S_{i}^{\prime}}^{\prime} \wedge N_{\sigma}\left(\left\|K_{X^{\prime}}+S_{i}^{\prime}+A_{q}^{\prime}+\Delta_{q}^{\prime}\right\|_{S_{i}^{\prime}}\right)
$$

This implies that $\sigma_{\mid S_{i}}^{l} \cdot s_{A \mid S_{i}}^{l m / q}$ extends to $X$ by the extension theorem (for example, see [[10], Theorem 3.4] or [[30], Theorem 6.3]), which in turn implies that $\log \left|\sigma_{\mid S_{i}}\right| \leq m \varphi_{q \mid S_{i}}$ where $\varphi_{q}$ is the maximal $\left(K_{X}+S+B+\frac{1}{q} A\right)$-psh function. Therefore we obtain that $\log \left|\sigma_{\mid S_{i}}\right| \leq m \varphi_{\mid S_{i}}$ by [[29], Proposition $6.11]$.

If we write $\mathfrak{b}_{k}=\mathcal{J}(k \varphi)$ and $\mathfrak{b}=\mathcal{O}_{X}(-m B)$, then we set an ideal $\mathcal{I}:=\mathcal{J}\left(\mathfrak{b}_{k, S}^{\frac{(1+\epsilon)(m-1)}{k}} \cdot \mathfrak{b}_{S}^{\frac{1}{m}}\right)$ for a sufficiently small number $\epsilon>0$ and every sufficiently divisible integer $k>0$. Note that $\mathcal{I}$ is well-defined on $S$ because $\mathcal{I}=\operatorname{Adj}_{S}((m-1) \varphi+\psi)_{S}$ where $\psi=\frac{1}{m} \log \left|s_{m B}\right|$ and $s_{m B}$ is the tautological section of $\mathcal{O}_{X}(-m B)$. Since

$$
\begin{aligned}
\log \left|\mathcal{I}_{S_{i}}\right| & =\log \left|\operatorname{Adj}_{\left.\left(S-S_{i}\right)\right|_{S_{i}}}\left((m-1) \varphi_{\mid S_{i}}+\psi_{\mid S_{i}}\right)\right| \\
& =\log \left|\operatorname{Adj}_{\left.\left(S-S_{i}\right)\right|_{S_{i}}}\left((m-1)(1+\epsilon) \varphi_{\mid S_{i}, k}+\psi_{\mid S_{i}}\right)\right| \geq \log \left|\sigma_{\mid S_{i}}\right|
\end{aligned}
$$


on every $S_{i}$, the section $\sigma$ vanishes along $\mathcal{I}$.

Step 2. One observes that the following exact sequence

$$
0 \longrightarrow \mathcal{J}((m-1) \varphi+\psi) \cdot \mathcal{O}_{X}(-S) \longrightarrow \operatorname{Adj}_{S}((m-1) \varphi+\psi) \longrightarrow \mathcal{I} \longrightarrow 0
$$

is exact. Since $\sigma \in H^{0}\left(S, m\left(K_{S}+\left.B\right|_{S}\right) \otimes \mathcal{I}\right)$, it suffices to show that the map

$$
\begin{aligned}
& H^{1}\left(X, \mathcal{O}_{X}\left(m\left(K_{X}+S+B\right)-S\right) \otimes \mathcal{J}((m-1) \varphi+\psi)\right) \\
& \quad \rightarrow H^{1}\left(X, \mathcal{O}_{X}\left(m\left(K_{X}+S+B\right)\right) \otimes \operatorname{Adj}_{S}((m-1) \varphi+\psi)\right)
\end{aligned}
$$

is injective. Since $K_{X}+S+B$ is abundant, by [29], Proposition 6.10, Proposition 6.11 and Corollary 6.13(5)] and Lemma 5.16 we choose a sufficiently divisible integer $k$ such that $\mathcal{J}(m \varphi+\psi)=\mathcal{J}\left(\mathfrak{a}_{k}^{\frac{m}{k}} \cdot \mathfrak{b}^{\frac{1}{m}}\right)$ and $\left.\operatorname{Adj}_{S}(m \varphi+\psi)\right)=\operatorname{Adj}_{S}\left(\mathfrak{b}_{k}^{\frac{(1+\epsilon) m}{k}} \cdot \mathfrak{b}^{\frac{1}{m}}\right)$ where $\mathfrak{a}_{k}=\mathfrak{b}\left(\left|k\left(K_{X}+S+B\right)\right|\right)$. Let $\pi: X^{\prime} \rightarrow X$ be a $\log$ resolution of $\mathfrak{a}_{k} \cdot \mathfrak{b}_{k} \cdot \mathfrak{b}$. If we write $\mathfrak{a}_{k} \cdot \mathcal{O}_{X^{\prime}}=$ $\mathcal{O}_{X^{\prime}}\left(-F_{k}\right), \mathfrak{b}_{k} \cdot \mathcal{O}_{X^{\prime}}=\mathcal{O}_{X^{\prime}}\left(-G_{k}\right)$ and $T:=\left\lfloor\frac{m-1}{k} F_{k}+B\right\rfloor-\left\lfloor\frac{m-1}{k} G_{k}+B\right\rfloor$, then we have a natural isomorphism

$$
\begin{aligned}
H^{1}\left(X^{\prime}, \mathcal{O}_{X^{\prime}}\left(K_{X^{\prime}}+\right.\right. & \left.\left.\frac{m-1}{k} M_{k}+\left\{\frac{m-1}{k} F_{k}+B\right\}\right)\right) \\
& \cong H^{1}\left(X, \mathcal{O}_{X}\left(m\left(K_{X}+S+B\right)-S\right) \otimes \mathcal{J}((m-1) \varphi+\psi)\right)
\end{aligned}
$$

where $M_{k}$ is a free divisor such that $\left|k \pi^{*}\left(K_{X}+S+B\right)\right|=\left|M_{k}\right|+F_{k}$, and an inclusion

$$
\begin{aligned}
& H^{1}(X,\left.\mathcal{O}_{X}\left(m\left(K_{X}+S+B\right)\right) \otimes \operatorname{Adj}_{S}((m-1) \varphi+\psi)\right) \\
& \hookrightarrow H^{1}\left(X^{\prime}, \mathcal{O}_{X^{\prime}}\left(K_{X^{\prime}}+S^{\prime}+(m-1) \pi^{*}\left(K_{X}+S+B\right)-\right.\right. \\
& \\
&\left.\left.\left\lfloor\frac{(1+\epsilon)(m-1)}{k} G_{k}+B\right\rfloor\right)\right)
\end{aligned}
$$

by the Leray spectral sequence and the exact sequence of low degrees.

Step 3. Note that the morphism

$$
\begin{aligned}
\iota: H^{1}\left(X^{\prime}, \mathcal{O}_{X^{\prime}}\left(K_{X^{\prime}}+\frac{m-1}{k} M_{k}+\left\{\frac{m-1}{k} F_{k}+B\right\}\right)\right) \\
\quad \longrightarrow H^{1}\left(X^{\prime}, \mathcal{O}_{X^{\prime}}\left(K_{X^{\prime}}+\frac{m-1}{k} M_{k}+\left\{\frac{m-1}{k} F_{k}+B\right\}+S^{\prime}+T\right)\right)
\end{aligned}
$$

factors through $H^{1}\left(X^{\prime}, \mathcal{O}_{X^{\prime}}\left(K_{X^{\prime}}+S^{\prime}+(m-1) \pi^{*}\left(K_{X}+S+B\right)-\right.\right.$ $\left.\left.\left\lfloor\frac{(1+\epsilon)(m-1)}{k} G_{k}+B\right\rfloor\right)\right)$. We write $T=\sum_{j=1}^{l} a_{j} T_{j}$. After reindexing we 
can assume that

$$
\operatorname{ord}_{T_{j}}\left(\left\lfloor\frac{m-1}{k} F_{k}+B\right\rfloor-\left\lfloor\frac{(1+\epsilon)(m-1)}{k} G_{k}+B\right\rfloor\right)>0
$$

for $1 \leq j \leq p$ and

$$
\operatorname{ord}_{T_{j}}\left(\left\lfloor\frac{m-1}{k} F_{k}+B\right\rfloor-\left\lfloor\frac{(1+\epsilon)(m-1)}{k} G_{k}+B\right\rfloor\right) \leq 0
$$

for $p<j \leq l$. It is easy to check that the morphism

$$
\begin{aligned}
& H^{1}\left(X^{\prime}, \mathcal{O}_{X^{\prime}}\left(K_{X^{\prime}}+\frac{m-1}{k} M_{k}+\left\{\frac{m-1}{k} F_{k}+B\right\}\right)\right) \\
& \quad \hookrightarrow H^{1}\left(X^{\prime}, \mathcal{O}_{X^{\prime}}\left(K_{X^{\prime}}+\frac{m-1}{k} M_{k}+\left\{\frac{m-1}{k} F_{k}+B\right\}+T^{\prime}\right)\right)
\end{aligned}
$$

is injective where $T^{\prime}$ is a reduced divisor which consists of those $T_{j}$ 's such that $1 \leq j \leq p$ and $T_{j} \nsubseteq \mathbb{S u p p}\left(\left\{\frac{m-1}{k} F_{k}+B\right\}\right)$ by the exact sequence of low degrees, and the morphism

$$
\begin{aligned}
\iota^{\prime}: & H^{1}\left(X^{\prime}, \mathcal{O}_{X^{\prime}}\left(K_{X^{\prime}}+\frac{m-1}{k} M_{k}+\left\{\frac{m-1}{k} F_{k}+B\right\}+T^{\prime}\right)\right) \\
& \longrightarrow H^{1}\left(X^{\prime}, \mathcal{O}_{X^{\prime}}\left(K_{X^{\prime}}+\frac{m-1}{k} M_{k}+\left\{\frac{m-1}{k} F_{k}+B\right\}+S^{\prime}+\sum_{j=1}^{p} a_{j} T_{j}\right)\right)
\end{aligned}
$$

factors through $H^{1}\left(X^{\prime}, \mathcal{O}_{X^{\prime}}\left(K_{X^{\prime}}+S^{\prime}+(m-1) \pi^{*}\left(K_{X}+S+B\right)-\right.\right.$ $\left.\left.\left\lfloor\frac{(1+\epsilon)(m-1)}{k} G_{k}+B\right\rfloor\right)\right)$. Therefore it suffices to prove that $\iota^{\prime}$ is injective. Since there exists an effective divisor $D \sim_{\mathbb{Q}} K_{X}+S+B$ such that $D$ contains $S$ in its support, we can assume that $\left(X^{\prime}, \frac{m-1}{k} M_{k}+\left\{\frac{m-1}{k} F_{k}+B\right\}+T^{\prime}\right)$ is $\log$ smooth and dlt such that $\operatorname{Supp}\left(S^{\prime}+\sum_{j=1}^{p} a_{j} T_{j}\right) \subseteq \operatorname{Supp}\left(\frac{m-1}{k} M_{k}+\left\{\frac{m-1}{k} F_{k}+B\right\}+T^{\prime}\right)$. Hence the injectivity of $\iota^{\prime}$ follows from [[22], Theorem 1.1] or [[1], Corollary 5.2].

Remark 5.19. It is conjectured that Proposition 5.18 holds without the assumption $K_{X}+S+B$ being abundant. Using analytic methods, J.-P. Demailly, C. D. Hacon and M. Păun proved this in [14] when $(X, S+B)$ is plt and there exists an effective divisor $D \sim_{\mathbb{Q}} K_{X}+S+B$ such that $S \subseteq \operatorname{Supp}(D) \subseteq \operatorname{Supp}(S+B)$. At this point we cannot attack similar problems without using complex analysis. However, the analytic argument fails in the dlt case because the $L^{2}$-estimates behave badly on the intersection of components of $S$. It is expected that we could combine algebraic and analytic methods to deal with these problems. It is also interesting that the proof 
above provides an essential application of the recent injectivity theorem (see [1] and [22]).

\section{References}

[1] F. Ambro; An injectivity theorem. Compositio Mathematica. Volume 150, Issue 06 (2014), 999-1023.

[2] C. Birkar, P. Cascini, C. D. Hacon and J. McKernan. Existence of minimal models for varieties of log general type. J. Amer. Math. Sci. 23 (2010), 405-468.

[3] S. Boucksom; Divisorial Zariski decompositions on compact complex manifolds. Ann. Sci. École Norm. Sup. 37 (2004), no. 4, 45-76.

[4] S. Boucksom, C. Favre, M. Jonsson; Valuations and plurisubharmonic singularities, Publ. Res. Inst. Math. Sci. 44 (2008),449-494.

[5] S. Boucksom, C. Favre, M. Jonsson; Singular semipositive metrics in non-Archimedean geometry. Preprint (2012) arXiv:1201.0187.

[6] S. Boucksom, C. Favre, M. Jonsson; Solution to a non-Archimedean Monge-Ampre equation. Preprint (2012) arXiv:1201.0188.

[7] S. Boucksom, C. Favre, M. Jonsson; A refinement of Izumi's Theorem. Preprint (2012) arXiv:1209.4104.

[8] S. Boucksom, T. de Fernex; C. Favre; The volume of an isolated singularity. Duke Math. J. Volume 161, Number 8 (2012), 1411-1612.

[9] S. Boucksom, T. de Fernex, C. Favre, S. Urbinati; Valuation spaces and multiplier ideals on singular varieties. Preprint (2013) arXiv:1307.0227.

[10] P. Cascini, V. Lazić; New outlook on the minimal model program, I Duke Math. J. Volume 161, Number 12 (2012), 2415-2467.

[11] J.-P. Demailly; Regularization of closed positive currents and intersection theory. J. Alg. Geom. 1 (1992), 361-409.

[12] J.-P. Demailly; A numerical criterion for very ample line bundles J. Differential. Geom. 37 (1993), 323-374.

[13] J.-P. Demailly, L. Ein, R. Lazarsfeld; A subadditivity property of multiplier ideals. Michigan Math. J. 48 (2000), 137-156. 
[14] J.-P. Demailly, C. D. Hacon, M. Păun; Extension theorems, Nonvanishing and the existence of good minimal models. Acta Mathematica Volume 210, Issue 2 (2013), 203-259.

[15] J.-P. Demailly, J. Kollar; Semi-continuity of complex singularity exponents and Kähler-Einstein metrics on Fano orbifolds. Annales Scientifiques de l'école Normale Supérieure Volume 34, Issue 4 (2001), 525556.

[16] L. Ein, R. Lazarsfeld, M. Mustaţă, M. Nakamaye, M. Popa; Asymptotic invariants of base loci. Ann. Inst. Fourier. 56 no.6 (2006), 1701-1734.

[17] L. Ein, R. Lazarsfeld, M. Mustaţă, M. Nakamaye, M. Popa; Restricted volumes and base loci of linear series. Amer. J. Math. 131 (2009), no. $3,607-651$.

[18] L. Ein, M. Mustaţă; Inversion of adjunction for local complete intersection varieties. American Journal of Mathematics. Vol. 126, No. 6 (2004), 1355-1365.

[19] L. Ein, M. Mustaţă, T. Yasuda; Jet schemes, log discrepancies and inversion of adjunction. Inventiones mathematicae. Volume 153, Issue 3 (2003), 519-535.

[20] L. Ein, M. Popa; Global division of cohomology classes via injectivity. arXiv:0712.3186. To appear in the Michigan Math. J. volume in honor of Mel Hochster's 65th birthday.

[21] O. Fujino; Introduction to the log minimal model program for log canonical pairs. preprint (2009). arXiv:0907.1506v1.

[22] O. Fujino; Injectivity theorems. Preprint (2013) arXiv:1303.2404.

[23] O. Fujino, Y. Gongyo; Log pluricanonical representations and abundance conjecture. Compositio Mathematica. Volume 150, Issue 04 (2014), 593-620.

[24] C. Favre, M. Jonsson; The valuative tree. Lecture Notes in Mathematics 1853, Springer, 2004.

[25] C. Favre, M. Jonsson; Valuative analysis of planar plurisubharmonic functions, Invent. Math. 162 (2005), no. 2, 271-311

[26] C. Favre, M. Jonsson; Valuations and multiplier ideals. J. Amer. Math. Soc. 18 (2005), 655-684. 
[27] Q. Guan, X. Zhou; Strong openness conjecture and related problems for plurisubharmonic functions. arXiv:1401.7158.

[28] C. D. Hacon; On the log canonical inversion of adjunction. Proceedings of the Edinburgh Mathematical Society (Series 2). Volume 57, Issue 01 (2014), 139-143.

[29] Z. Hu; Valuative multiplier ideals. Pacific Journal of Mathematics 270-1 (2014), 95-128.

[30] C. D. Hacon; J. Mckernan Existence of minimal models for varieties of log general type II. J. Am. Math. Soc 23(2010), 469-490

[31] M. Jonsson, M. Mustaţă: Valuations and asymptotic invariants for sequences of ideals. arXiv: 1011.3699. To appear in Ann. Inst. Fourier (Grenoble).

[32] M. Jonsson, M. Mustaţă: An algebraic approach to the openness conjecture of Demailly and Kollar. arXiv:1205.4273. To appear in J. Inst. Math. Jussieu.

[33] M. Kawakita; Inversion of adjunction of log canonicity. Inventiones mathematicae. Volume 167, Issue 1 (2007), 129-133.

[34] J. Kollár; Lectures on resolution of singularities. volume 166 of Annals of Mathematics Studies. Princeton University Press, Princeton, NJ, 2007.

[35] J. Kollár, S. Mori; Birational Geometry of Algebraic Varieties. Cambridge University Press (1998).

[36] R. Lazarsfeld: Positivity in algebraic geometry. II. Positivity of vector bundles and multiplier ideals Ergebnisse der Mathematik und ihrer Grenzgebiete, 49. Springer-Verlag, Berlin, 2004.

[37] B. Lehmann; Algebraic bounds on analytic multiplier ideals. Preprint (2011) arXiv: 1109.4452.

[38] L. Lempert; Modules of square integrable holomorphic germs. Preprint (2014) arXiv: 1404.0407.

[39] M. Mustata, J. Nicaise; Weight functions on non-archimedean analytic spaces and the Kontsevich-Soibelman skeleton. Preprint (2012) arXiv:1212.6328.

[40] N. Nakayama; Zariski decomposition and abundance. MSJ Memoirs 14, Tokyo (2004). 
[41] J. Nicaise, C. Xu; The essential skeleton of a degeneration of algebraic varieties. Preprint (2013) arXiv:1307.4041.

[42] M. Păun; Relative critical exponents, non-vanishing and metrics with minimal singularities. Inventiones mathematicae Volume 187, Issue 1, 195-258.

[43] V. Shokurov; 3-fold log flips. Russian Acad. Sci. Izvestiya Mathematics(1993),40(1):95

[44] S. Takagi; Formulas for multiplier ideals on sigular varieties. American Journal of Mathematics. Volume 128, No. 6 (2006), 1345-1362.

[45] S. Takagi; Adjoint ideals along closed subvarieties of higher codimension. Journal für die reine und angewandte Mathematik (Crelles Journal). Volume 2010, Issue 641, 145-162.

[46] M. Temkin; Metrization of differential pluriforms on Berkovich analytic spaces. Preprint (2014) arXiv:1410.3079.

Zhengyu $\mathrm{Hu}$

Center of Mathematical Sciences,

Zhejiang University,

38 Zheda Road, Hangzhou, 310013,

China

E-mail: zhengyuhu16@gmail.com 